DRAFT VERSION OCTOBER 13, 2018

Typeset using LATEX twocolumn style in AASTeX61

\title{
IS THERE A CIRCUMBINARY PLANET AROUND NSVS 14256825?
}

\begin{abstract}
Ilham Nasiroglu, ${ }^{1}$ Krzysztof Goździewski, ${ }^{2}$ Aga SŁowikowska, Krzysztof Krzeszowski, Michą Żejmo, ${ }^{3}$ Staszek Zola, ${ }^{4}$ Huseyin ER, ${ }^{1}$
\end{abstract} Waldemar Ogłoza, Marek Dróżdż, ${ }^{5}$ Dorota Koziel-Wierzbowska, Bartlomiej Debski, ${ }^{6}$ and Nazli Karaman ${ }^{7}$

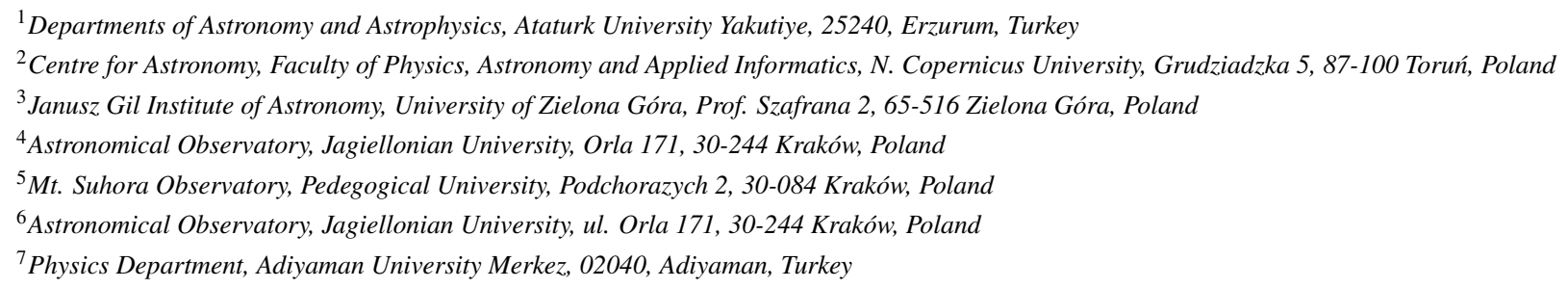

(Received December 7, 2016; Revised January 6, 2017; Accepted October 13, 2018)

Submitted to ApJ

\begin{abstract}
The cyclic behaviour of $(\mathrm{O}-\mathrm{C})$ residuals of eclipse timings in the sdB+M eclipsing binary NSVS 14256825 was previously attributed to one or two Jovian-type circumbinary planets. We report 83 new eclipse timings that not only fill in the gaps in those already published but also extend the time span of the $(\mathrm{O}-\mathrm{C})$ diagram by three years. Based on the archival and our new data spanning over more than 17 years we re-examined the up to date system $(\mathrm{O}-\mathrm{C})$. The data revealed systematic, quasi-sinusoidal variation deviating from an older linear ephemeris by about $100 \mathrm{~s}$. It also exhibits a maximum in the (O-C) near JD 2,456,400 that was previously unknown. We consider two most credible explanations of the $(\mathrm{O}-\mathrm{C})$ variability: the light propagation time due to the presence of an invisible companion in a distant circumbinary orbit, and magnetic cycles reshaping one of the binary components, known as the Applegate or Lanza-Rodonó effect. We found that the latter mechanism is unlikely due to the insufficient energy budget of the M-dwarf secondary. In the framework of the third-body hypothesis, we obtained meaningful constraints on the Keplerian parameters of a putative companion and its mass. Our best-fitting model indicates that the observed quasi-periodic (O-C) variability can be explained by the presence of a brown dwarf with the minimal mass of 15 Jupiter masses rather than a planet, orbiting the binary in a moderately elliptical orbit $(e \simeq 0.175)$ with the period of $\sim 10$ years. Our analysis rules out two planets model proposed earlier.
\end{abstract}

Keywords: Stars: binaries: eclipsing - Stars: binaries: close - Stars: subdwarfs - Stars: individual: NSVS 14256825- Planetary systems: detection. 


\section{INTRODUCTION}

The number of discovered exoplanets around binary systems increases rapidly. These discoveries have sparked a rising interest on this subject among researchers, and consequently, they drive the development of new detection techniques. Studies of circumbinary planets (CBPs) have taken us much closer toward answering the fundamental questions how such planets form and evolve. The properties of circumbinary planets are likely different than these orbiting isolated stars (Lee et al. 2009).

The most remarkable discovery made with the transit method with the Kepler satellite is the discovery of a circumbinary planet transiting across both stars of the close binary system Kepler-16 (AB) (Doyle et al. 2011). The transits in this system leave no doubt about the existence of planets in the so-called "P-type" orbits, i.e. circumbinary orbits. So far the longest-period transiting CBP is Kepler-1647 with the orbital period of $\sim 1100$ days (Kostov et al. 2016b).

Even before the Kepler discoveries, timing observations have provided evidence of planets orbiting binary systems. Hints for such companions were reported by Deeg et al. (2008) and Lee et al. (2009) for the eclipsing binaries CM Dra and HW Vir, respectively. The presence of these objects are indicated through the Light Travel Time (LTT) effect indicated by the variations in the timings of eclipse minima w.r.t. the linear ephemeris $(\mathrm{O}-\mathrm{C})$. Quasi-periodic variations of the $(\mathrm{O}-\mathrm{C})$ can result from the gravitational tug due to distant planets (companions), which leads to swinging of the eclipsing binary, and causing the eclipses to appear slightly earlier or later w.r.t the linear ephemeris. The LTT effect can be measured with a high accuracy and used to infer the presence of planetary-mass companions around binary stars (Irwin 1952; Horner et al. 2012; Goździewski et al. 2012, 2015). In contrast to other techniques, the timing method is sensitive to massive extrasolar planets in long-period orbits. Futhermore, for low-mass binaries the amplitude of the LTT effect increases (Ribas 2005; Pribulla et al. 2012).

Recently, a number of planetary mass companions orbiting the cataclysmic variables (CVs) and post-common envelope binaries (PCEBs) have been reported. For instance, two planets for NN Ser (Beuermann et al. 2010) and UZ For (Potter et al. 2011), a single planet for DP Leo (Beuermann et al. 2011) and V470 Cam (Beuermann et al. 2012b) have been claimed. A long-term stable system of three planets hosted by $\mathrm{HU}$ Aqr, with the middle one being on a retrograde orbit, was recently proposed by Goździewski et al. (2015).

NSVS 14256825 was discovered through the Northern Sky Variability Survey (NSVS) (Woźniak et al. 2004a). Wils et al. (2007) identified this system as an eclipsing binary with an amplitude of variations in the range of 13.22-14.03 (V). These authors also presented the first $\mathrm{B}, \mathrm{V}, \mathrm{I}_{\mathrm{c}}$ light curves and physical parameters of this binary $(\mathrm{P}=0.110374230(2)$ days $)$, along with a few eclipse times. NSVS 14256825 is a member of the HW Vir family (PCEB) consisting of a OB subdwarf and a M dwarf companion ( $\mathrm{sdOB}+\mathrm{dM}$ ) (Almeida et al. 2012). The following physical and geometrical parameters were obtained: $\mathrm{i}=82 .^{\circ} 5 \pm 0 .^{\circ} 3$ (inclination of the system), $\mathrm{M}_{1}=0.419 \pm 0.070 \mathrm{M}_{\odot}, \mathrm{R}_{1}=0.188 \pm 0.010 \mathrm{R}_{\odot}, \mathrm{M}_{2}=0.109$ $\pm 0.023 \mathrm{M}_{\odot} \mathrm{R}_{2}=0.162 \pm 0.008 \mathrm{R}_{\odot}$ (the masses and radii of the components), $a=0.80 \pm 0.04 \mathrm{R}_{\odot}$ (separation between the components) from the photometric and spectroscopic observations (Almeida et al. 2012).

The eclipse times of NSVS 14256825 have been reported by Wils et al. (2007); Kilkenny \& Koen (2012); Beuermann et al. (2012b); Almeida et al. (2013) and Lohr et al. (2014). Qian et al. (2010) and Zhu et al. (2011) also argued for a $\mathrm{O}-\mathrm{C}$ cyclic variation, however they have not published any supporting data yet. Kilkenny \& Koen (2012), reported an increasing orbital period of this system with a rate of $\sim 1.1 \times 10^{-10} \mathrm{ss}^{-1}$. Beuermann et al. (2012b) detected cyclic period changes and suggested the presence of a single circumbinary planet of $\sim 12 \mathrm{M}_{\text {Jup }}$ with a period of $\sim 20 \mathrm{yrs}$. Almeida et al. (2013) presented a few additional eclipse times and argued for the presence of two circumbinary planets with periods of $\sim 3.5 \mathrm{yrs}$ and $\sim 6.7 \mathrm{yrs}$, and masses of $\sim 3 \mathrm{M}_{\text {Jup }}$ and $\sim 8.0 \mathrm{M}_{\mathrm{Jup}}$, respectively. Wittenmyer et al. (2013) presented a dynamical analysis of the orbital stability of the two planet model proposed by Almeida et al. (2013). They found that this model is extremely unstable on time scale of less than a thousand years. Moreover, Hinse et al. (2014) also performed a detailed data analysis of the timing measurements of this system. They concluded that the time span of eclipse time variations is not long enough neither to explain any particular one-planet model nor provide a convincing evidence for a second planetary companion. Recently, Lohr et al. (2014) presented many new eclipse times of NSVS 14256825 from the SuperWASP archive. Their measurements obtained between 2006 and 2011 confirm the overall trend already seen in the $\mathrm{O}-\mathrm{C}$ diagram.

In this study, we present 83 new mid-eclipse times of NSVS 14256825 obtained between 2009-08-21 and 201611-03 that together with the literature data give 153 eclipses over the time span of 17 years. We combined our new data with the previously published measurements to analyse the orbital period variations of this system. In Section 2 we present the observations and data reduction process together with the methodology used to obtain the eclipse times. Section 3 presents the procedure applied to examine the period variations, while the results are gathered in Section 4. In Sections 5 and 6 we discuss and conclude our findings. We include some additional materials in the on-line Appendix.

\section{NEW PHOTOMETRY OF NSVS 14256825}


We performed photometric observations of NSVS 14256825 between 2009-08-21 and 2016-11-03 with five different telescopes: the $1.3 \mathrm{~m}$ telescope at the Skinakas Observatory (SKO, Creete, Greece), the $0.5 \mathrm{~m}$ telescope at the Astronomical Observatory of the Jagiellonian University (KRK, Kraków, Poland), the $0.6 \mathrm{~m}$ telescope at the Mt. Suhora Observatory (SUH, Koninki, Poland), the $0.6 \mathrm{~m}$ telescope at the Adiyaman University Observatory (ADYU60, Adiyaman, Turkey) and with the $1 \mathrm{~m}$ telescope at the TUBITAK National Observatory (TUG, Antalya, Turkey).

Between 2009 and 2013, observations were performed using the SKO, KRK and SUH telescopes, while these taken between 2014 and 2016, with the ADYU60 and TUG telescopes. We gathered data with the following CCD cameras: the Andor iKon DZ-936B-BV (KRK), the Andor DZ436 (SKO), the Apoge Alta U47 (SUH), the Andor iKon-M934 (ADYU60) and the SI1100 (TUG). A summary of observations is given in Tab. 3, where the start observing date, the cycle number, eclipse type (primary - 1 , secondary - 2), filter used, exposure time and readout time are listed.

The CCD data were reduced with the pipeline developed using Python, IRAF and Sextractor software. The usual bias and dark subtraction as well as flat-field correction were applied to all images. For Andor CCDs dark counts were negligible and therefore, only bias subtraction was done. A nearby constant star in the field of view, comparable to the target star in brightness and color, was chosen as the comparison star. Since our major goal was to obtain differential photometry only, we did not observe any photometric standard stars. For each night a light curve was constructed consisting of extracted magnitude differences and time in the form of JD UTC. The mid-exposure times were taken.

We modelled the shapes of the eclipses with a modified and truncated inverted Gaussian $G(\tau)$ multiplied by a polynomial, as described in Section 2. of Beuermann et al. (2012b). The model involves 8 parameters, including eclipse minimum time $\left(T_{\text {obs }}\right)$ denoted as $p_{1}$ in Beuermann et al. (2012b). The resulting parameters values and their respective uncertainties (including $\sigma_{T_{\mathrm{obs}}}$ ) were obtained from the fitting procedure as a term of the resulting covariance matrix from the nonlinear least squares fitting algorithm. Figs. 8 and 9 show the observed light curves and model fits. The derived eclipse times were converted to the barycentric dynamical time (BJD), using the FK5 sky coordinates of NSVS $14256825\left(\alpha=20^{\mathrm{h}} 20^{\mathrm{m}} 00^{\mathrm{s}} .458, \delta=+04^{\circ} 37^{\prime} 56^{\prime \prime} .50\right)$ and the geodetic coordinates of each given observatory, with the help of the numerical procedure developed by Eastman et al. (2010). The eclipse minimum times, together with their respective errors, obtained from our new measurements are listed in Tab. 1, while all timings (including these published in the literature) are gathered in Tab. 4 (available in the online version only). The cycle numbers in Tabs. 1 and 4 are given according to the ephemeris from Beuermann et al. (2012b). We would like to note that we have three pairs of simultaneous observations of NSVS 14256825 with the TUG and Adiyaman telescopes, i.e. these for cycle numbers: 30669, 30670 and 30931. For each pair the difference between derived $T_{\text {obs }}$ agrees within their errors derived from the fit, i.e. less than two seconds.

\section{LTT MODELS OF THE (O-C)}

To model the eclipses ephemeris with the presence of a hypothetical third body we used the following formulae

$$
T_{\mathrm{eph}}(L)=t_{0}+P_{\mathrm{bin}} L+\gamma_{\mathrm{tb}}(L),
$$

where $\gamma_{\mathrm{tb}}$ represents the Light Travel Time (LTT) term (Irwin 1952). In our formulation this term is parametrised by the Keplerian orbital elements of the third body companion in orbit around the mass center of the binary (Goździewski et al. 2012):

$$
\gamma_{\mathrm{tb}}(t)=K\left[\sin \omega(\cos E(t)-e)+\cos \omega \sqrt{1-e^{2}} \sin E(t)\right],
$$

where $K$ is the semi-amplitude of the LTT signal, $e, \omega, P, \tau$ are the eccentricity, periastron argument, orbital period and the time of periastron passage of the relative orbit of the putative companion w.r.t. the binary. We note that $P$ and $\tau$ are introduced indirectly through the Kepler equation

$$
\frac{2 \pi}{P}(t-\tau)=E-e \sin E .
$$

Due to very different time scales of orbital motion, the binary is represented as a point with the total mass of both stellar components equal to $0.528 \mathrm{M}_{\odot}$ (Almeida et al. 2012). Furthermore, to account for small eccentricity, we introduce Poincaré elements $(x \equiv e \cos \omega, y \equiv e \sin \omega$ ) which make it possible to get rid of weakly constrained eccentricity and pericenter argument $\omega$ for quasi-circular and moderately eccentric orbits.

To express the $(\mathrm{O}-\mathrm{C})$ variability through $\gamma_{\mathrm{pl}}(L(t))$, we optimize the likelihood function $\mathcal{L}$,

$$
\log \mathcal{L}(\mathcal{D} \mid \xi)=-\frac{1}{2} \chi^{2}-\frac{1}{2} \sum_{i} N \log \sigma_{i}^{2}-\frac{1}{2} N \log 2 \pi,
$$

where the $\chi^{2}$ function

$$
\chi^{2}(\mathcal{D}, \boldsymbol{\xi})=\sum_{i}^{N} \frac{[\mathrm{O}(\mathcal{D})-\mathrm{C}(\boldsymbol{\xi})]_{i}^{2}}{\sigma_{i}^{2}}
$$

depends on model parameters through $(\mathrm{O}-\mathrm{C})_{i} \equiv\left(T_{\text {obs }}\left(L_{i}\right)-\right.$ $\left.T_{\text {eph }}\left(L_{i}\right)\right)$ and the measurements uncertainties $\sigma_{i}$, where $i=$ $1 \ldots, N$ (Goździewski et al. 2015). Here, $(\mathrm{O}-\mathrm{C})_{i}$ denotes the deviation of the observed $i$-th eclipse time-mark from its BJD ephemeris (Eq. 1) for cycle $L_{i} \equiv L\left(t_{i}\right)$. The model parameters 
Table 1. List of new NSVS 14256825 eclipse times. Cycle number, time of the minimum, its error, type of the eclipse (1 - primary, 2 - secondary) and references are given. References are for the observatories: (5) the Astronomical Obs. of the Jagiellonian Univ., (6) the Mt. Suhora Obs., (7) the Skinakas Obs., (8) the TUBITAK National Obs. and (9) the Adiyaman Univ. Obs.

\begin{tabular}{|c|c|c|c|c|}
\hline Cycle & BJD & Error [days] & Eclipse Type & Ref \\
\hline 7167.5 & 2455065.315208 & 0.000082 & 2 & 5 \\
\hline 7204.0 & 2455069.343870 & 0.000036 & 1 & 5 \\
\hline 7223.0 & 2455071.440996 & 0.000014 & 1 & 5 \\
\hline 7386.0 & 2455089.432002 & 0.000024 & 1 & 6 \\
\hline 7503.0 & 2455102.345843 & 0.000018 & 1 & 5 \\
\hline 7557.0 & 2455108.306027 & 0.000041 & 1 & 5 \\
\hline 7955.0 & 2455152.234856 & 0.000018 & 1 & 6 \\
\hline 9797.0 & 2455355.544034 & 0.000035 & 1 & 5 \\
\hline 10593.0 & 2455443.401924 & 0.000010 & 1 & 6 \\
\hline 10918.0 & 2455479.273564 & 0.000012 & 1 & 6 \\
\hline 14162.0 & 2455837.327516 & 0.000016 & 1 & 6 \\
\hline 16808.0 & 2456129.377577 & 0.000015 & 1 & 7 \\
\hline 16808.5 & 2456129.432822 & 0.000023 & 2 & 7 \\
\hline 16817.0 & 2456130.370981 & 0.000006 & 1 & 7 \\
\hline 16835.0 & 2456132.357723 & 0.000017 & 1 & 6 \\
\hline 16835.5 & 2456132.412907 & 0.000033 & 2 & 6 \\
\hline 17650.0 & 2456222.312685 & 0.000020 & 1 & 5 \\
\hline 17867.0 & 2456246.263846 & 0.000021 & 1 & 5 \\
\hline 19301.0 & 2456404.540320 & 0.000024 & 1 & 6 \\
\hline 19545.0 & 2456431.471682 & 0.000019 & 1 & 6 \\
\hline 19744.0 & 2456453.436075 & 0.000040 & 1 & 6 \\
\hline 19744.5 & 2456453.491275 & 0.000099 & 2 & 6 \\
\hline 20206.0 & 2456504.428991 & 0.000015 & 1 & 6 \\
\hline 20206.5 & 2456504.484030 & 0.000044 & 2 & 6 \\
\hline 20269.0 & 2456511.382498 & 0.000014 & 1 & 6 \\
\hline 20360.0 & 2456521.426538 & 0.000013 & 1 & 6 \\
\hline 20803.0 & 2456570.322299 & 0.000098 & 1 & 6 \\
\hline 20812.0 & 2456571.315608 & 0.000025 & 1 & 6 \\
\hline 20812.5 & 2456571.370781 & 0.000113 & 2 & 6 \\
\hline 20813.0 & 2456571.425973 & 0.000046 & 1 & 6 \\
\hline 20830.0 & 2456573.302321 & 0.000014 & 1 & 6 \\
\hline 20975.0 & 2456589.306568 & 0.000016 & 1 & 5 \\
\hline 23458.0 & 2456863.365367 & 0.000033 & 1 & 8 \\
\hline 23467.0 & 2456864.358634 & 0.000032 & 1 & 8 \\
\hline 24553.0 & 2456984.224844 & 0.000017 & 1 & 8 \\
\hline 26132.0 & 2457158.505405 & 0.000012 & 1 & 9 \\
\hline 26141.0 & 2457159.498659 & 0.000030 & 1 & 9 \\
\hline 26205.0 & 2457166.562708 & 0.000012 & 1 & 8 \\
\hline 26213.0 & 2457167.445649 & 0.000021 & 1 & 8 \\
\hline
\end{tabular}

Table 1. continued...

\begin{tabular}{|c|c|c|c|c|}
\hline Cycle & BJD & Error [days] & Eclipse Type & Ref \\
\hline 26331.0 & 2457180.469794 & 0.000026 & 1 & 9 \\
\hline 26413.0 & 2457189.520546 & 0.000020 & 1 & 8 \\
\hline 26422.0 & 2457190.513847 & 0.000010 & 1 & 8 \\
\hline 26422.5 & 2457190.569067 & 0.000075 & 2 & 8 \\
\hline 26648.0 & 2457215.458344 & 0.000012 & 1 & 9 \\
\hline 26683.0 & 2457219.321420 & 0.000012 & 1 & 8 \\
\hline 26684.0 & 2457219.431837 & 0.000013 & 1 & 8 \\
\hline 26730.0 & 2457224.509018 & 0.000014 & 1 & 9 \\
\hline 26846.0 & 2457237.312397 & 0.000017 & 1 & 9 \\
\hline 26911.0 & 2457244.486749 & 0.000012 & 1 & 9 \\
\hline 26937.5 & 2457247.411658 & 0.000056 & 2 & 8 \\
\hline 27009.0 & 2457255.303376 & 0.000015 & 1 & 9 \\
\hline 27073.0 & 2457262.367301 & 0.000016 & 1 & 9 \\
\hline 27082.0 & 2457263.360683 & 0.000034 & 1 & 9 \\
\hline 27099.0 & 2457265.237010 & 0.000020 & 1 & 9 \\
\hline 27154.0 & 2457271.307578 & 0.000031 & 1 & 9 \\
\hline 27408.0 & 2457299.342580 & 0.000028 & 1 & 9 \\
\hline 27543.0 & 2457314.243090 & 0.000010 & 1 & 9 \\
\hline 28004.0 & 2457365.125453 & 0.000022 & 1 & 9 \\
\hline 29411.5 & 2457520.476902 & 0.000061 & 2 & 8 \\
\hline 29412.0 & 2457520.532048 & 0.000015 & 1 & 8 \\
\hline 29611.0 & 2457542.496481 & 0.000012 & 1 & 9 \\
\hline 29620.0 & 2457543.489845 & 0.000014 & 1 & 9 \\
\hline 29647.0 & 2457546.469948 & 0.000039 & 1 & 9 \\
\hline 29956.0 & 2457580.575537 & 0.000013 & 1 & 8 \\
\hline 30135.0 & 2457600.332481 & 0.000014 & 1 & 9 \\
\hline 30163.0 & 2457603.422952 & 0.000012 & 1 & 9 \\
\hline 30172.0 & 2457604.416311 & 0.000011 & 1 & 9 \\
\hline 30180.0 & 2457605.299328 & 0.000013 & 1 & 9 \\
\hline 30397.0 & 2457629.250499 & 0.000009 & 1 & 8 \\
\hline 30399.0 & 2457629.471256 & 0.000020 & 1 & 8 \\
\hline 30408.0 & 2457630.464585 & 0.000006 & 1 & 8 \\
\hline 30660.0 & 2457658.278862 & 0.000008 & 1 & 9 \\
\hline 30669.0 & 2457659.272248 & 0.000008 & 1 & 9 \\
\hline 30669.0 & 2457659.272263 & 0.000014 & 1 & 8 \\
\hline 30670.0 & 2457659.382587 & 0.000009 & 1 & 8 \\
\hline 30670.0 & 2457659.382609 & 0.000015 & 1 & 9 \\
\hline 30705.0 & 2457663.245692 & 0.000008 & 1 & 9 \\
\hline 30714.0 & 2457664.239053 & 0.000010 & 1 & 9 \\
\hline 30904.0 & 2457685.210139 & 0.000012 & 1 & 9 \\
\hline 30931.0 & 2457688.190200 & 0.000015 & 1 & 8 \\
\hline 30931.0 & 2457688.190212 & 0.000011 & 1 & 9 \\
\hline 30941.0 & 2457689.293987 & 0.000027 & 1 & 9 \\
\hline 31004.0 & 2457696.247504 & 0.000009 & 1 & 8 \\
\hline
\end{tabular}


vector $\boldsymbol{\xi} \equiv\left(K, P, e, \omega, \tau, P_{b i n}, t_{0}, \sigma_{f}\right)$, and $N$ denotes the number of measurements encoded as data set $\mathcal{D}$. We note that all parameters of $T_{\text {eph }}$ are optimized. This more general form of $\mathcal{L}$ makes it also possible to determine the free parameter $\sigma_{f}$ that scales the raw uncertainties $\sigma_{i}$ in quadrature, such that $\sigma_{i, t}^{2} \rightarrow \sigma_{i}^{2}+\sigma_{f}^{2}$ results in $\chi_{v}^{2} \equiv \chi^{2} /(N-\operatorname{dim} \boldsymbol{\xi}) \sim 1$.

Optimisation of the dynamical model relies on investigating the space of 8 free model parameters $\boldsymbol{\xi}$, through sampling the posterior probability distribution $\mathcal{P}(\boldsymbol{\xi} \mid \mathcal{D})$ of the parameters $\boldsymbol{\xi}$, given the data set $\mathcal{D}: \mathcal{P}(\boldsymbol{\xi} \mid \mathcal{D}) \propto \mathcal{P}(\boldsymbol{\xi}) \mathcal{P}(\mathcal{D} \mid \boldsymbol{\xi})$, where $\mathcal{P}(\boldsymbol{\xi})$ is the prior, and the sampling data distribution $\mathcal{P}(\mathcal{D} \mid \boldsymbol{\xi}) \equiv$ $\log \mathcal{L}(\mathcal{D} \mid \boldsymbol{\xi})$. For all of these parameters, priors have been set as uniform (or uniform improper) through imposing parameters ranges available for the exploration, i.e., $K, P, \tau>0 \mathrm{~d}$, $\sigma_{f}>0 \mathrm{~d}$, and $x, y \in[-0.71,0.71], P_{\text {bin }} \in[0.110,0.112] \mathrm{d}$, and $\Delta t_{0} \in[-0.1,0.1] \mathrm{d}$ which is for the displacement w.r.t. the cycle $L=0$ for the epoch of $T_{0}=$ BJD 2455793.840061.

We sampled the posterior with the Markov Chain Monte Carlo (MCMC) emcee package of the affine-invariant ensemble sampler (Goodman \& Weare 2010), kindly provided by Foreman-Mackey et al. (2013).

\section{LTT MODEL RESULTS}

Due to the non-homogeneous timing data which are gathered across literature and in this manuscript, we consider three datasets. Dataset A includes all observations available to date, which encompasses CCD observations and 5 measurements from the NSVS and ASAS archives in Beuermann et al. (2012b), SuperWASP-derived timing data (Lohr et al. 2014), as well as our new measurements listed in Table 1. Due to a large scatter and uncertainties, the SuperWASP measurements are finally excluded in Dataset B. In Dataset C we also excluded the NSVS and ASAS measurements due to uncertain derivation of these measurements, which is discussed in Sect. 5.1. Then we subsequently analysed Datasets A, B and $\mathrm{C}$ individually. These particular datasets are illustrated in the (O-C) diagrams in Figs. 1, 2 and 3, respectively.

In all figures included in this section, we marked with grey rectangles the time interval after the last epoch in (Hinse et al. 2014). i.e. August 2012, which indicates our new measurements. We may expect that the orbital period of a putative third object may be constrained and that changes conclusions of Hinse et al. (2014), who were able to see only an increase of the $(\mathrm{O}-\mathrm{C})$.

Parameters of the linear ephemeris for Dataset A are

$$
T_{\mathrm{eph}}(L)=\text { BJD 2455793.84004(2) + L 0.110374083(2) },
$$

for Dataset B the linear ephemeris is described through

$$
T_{\text {eph }}(L)=\text { BJD 2455793.84005(3) }+L 0.110374082(3),
$$

while that for Dataset $\mathrm{C}$ is

$$
T_{\text {eph }}(L)=\text { BJD 2455793.84005(3) }+L 0.110374082(3),
$$

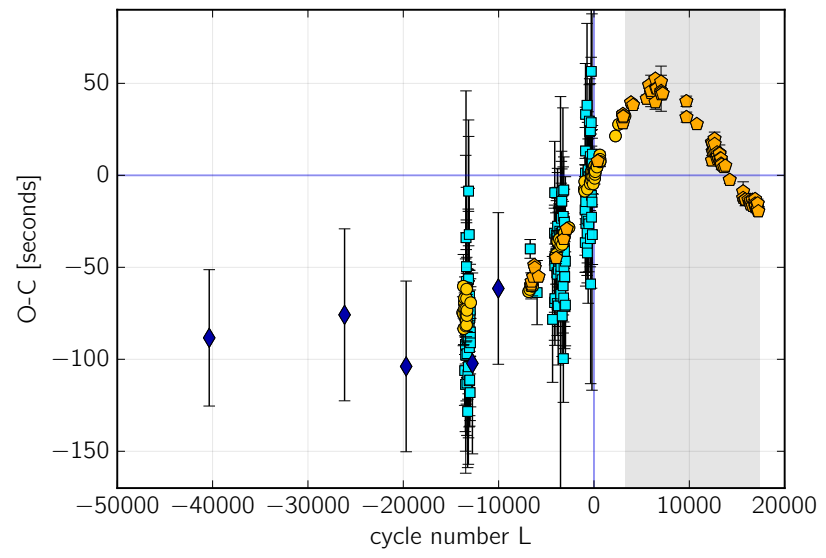

Figure 1. The (O-C) diagram w.r.t. the linear ephemeris for Dataset A, with all data available in the literature. Filled rectangles are for raw (unbinned) SuperWASP data, filled circles and diamonds are for other measurements prior to epoch of August 2012, and pentagons are for the new timing data in this paper (Tab. 1). See the text for details.

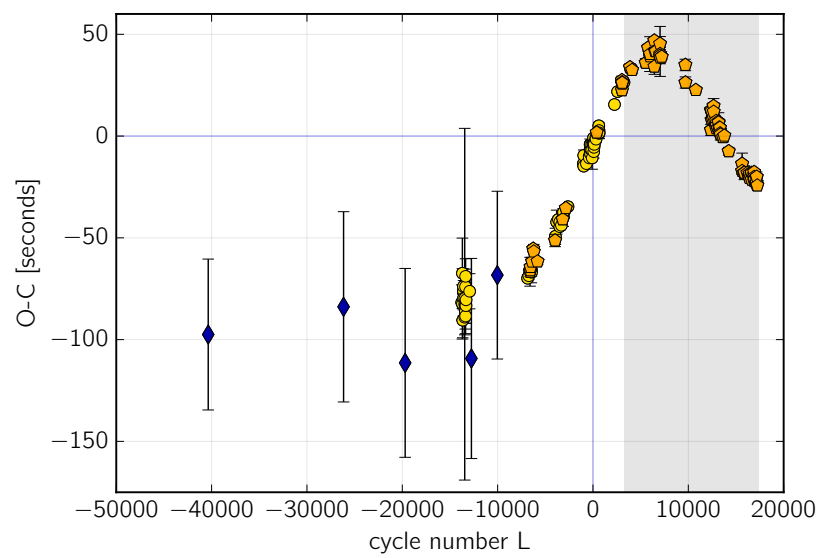

Figure 2. The $(\mathrm{O}-\mathrm{C})$ diagram w.r.t. the linear ephemeris for Dataset B. Dark blue diamonds are for the NSVS and ASAS measurements, filled circles are for data in the up-to date literature excluding SuperWASP measurements, dark diamonds are for NSVS and ASAS data, and darker pentagons are for the new measurements in this paper (Tab. 1). See the text for details.

where $T_{\text {eph }}$ stands here for the linear ephemeris of the BJD moment of the mid-eclipse of the cycle $L$. We chose the initial epoch $T_{0}$ of the cycle $L=0$ roughly in the middle of the observational window, i.e., $T_{0}=$ BJD 2455793.840061. It is clear that the linear ephemeris is essentially the same, within the errors at the last significant digit marked in brackets. However, the SuperWASP, NSVS and ASAS points strongly deviate from apparently a quasi-sinusoidal pattern formed by $(\mathrm{O}-\mathrm{C})$ derived from more accurate measurements.

Before sampling the posterior, which is determined through the likelihood function (Eq. 2), we first found the best-fitting parameters through maximising $\mathcal{L}$ with the genetic algorithm (Charbonneau 1995). Next we ran the 


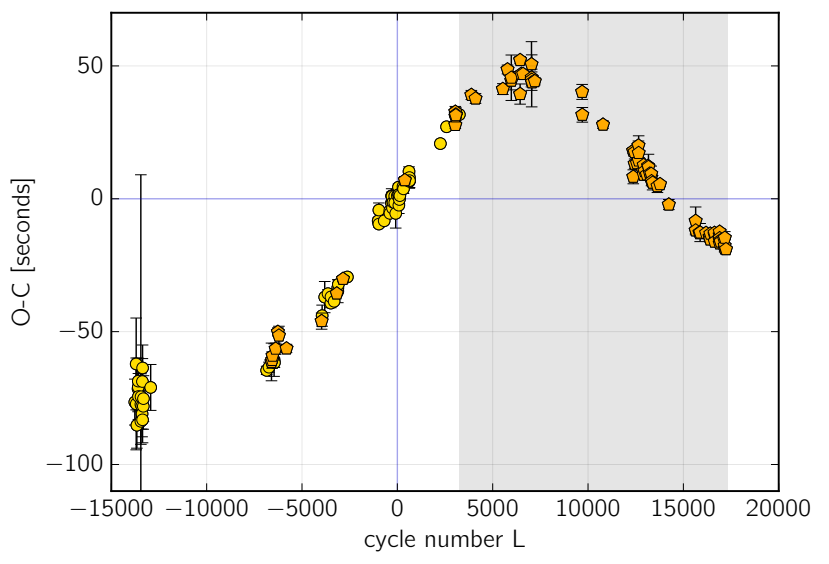

Figure 3. The $(\mathrm{O}-\mathrm{C})$ diagram w.r.t. the linear ephemeris for Dataset C. Filled circles are for data in the up-to date literature excluding SuperWASP, NSVS and ASAS measurements, and pentagons are for the new timing data in this paper (Tab. 1), see the text for details.

Table 2. Parameters of the third-body with linear ephemeris for Dataset C (Figs. 6 and 7). Parameter $\Delta t_{0}$ is for the shift relative to the observational middle-window epoch $T_{0}=$ BJD 2455793.840061. Total mass of the binary is $0.528 M_{\odot}$ (Almeida et al. 2012), see the text for details.

\begin{tabular}{lllc}
\hline Parameter & Value & $+\sigma$ & $-\sigma$ \\
\hline$K[\mathrm{~s}]$ & 48.9 & 1.6 & 1.2 \\
$P[$ day $]$ & 3632.8 & 169.6 & 131.7 \\
$x$ & 0 & 0.045 & 0.042 \\
$y$ & 0.175 & 0.032 & 0.031 \\
$\tau$ [day] & 7938.5 & 246.5 & 161.8 \\
$P_{\text {bin }}[$ day $]$ & 0.110374099 & $2 \times 10^{-9}$ & $3 \times 10^{-9}$ \\
$\Delta t_{0}[$ day $]$ & $-5 \times 10^{-5}$ & $2 \times 10^{-5}$ & $2 \times 10^{-5}$ \\
$\sigma_{f}[\mathrm{~s}]$ & 1.8 & 0.2 & 0.2 \\
mass $\left[\mathrm{M}_{\text {Jup }}\right]$ & 14.75 & 0.13 & 0.13 \\
$a$ [au $]$ & 3.74 & 0.12 & 0.09 \\
$e$ & 0.175 & 0.012 & 0.003 \\
$\omega[$ deg] & 90.11 & 15.37 & 12.89 \\
\hline
\end{tabular}

MCMC sampler for 512 initial conditions inside a small ball centered on this solution. We tested chain lengths from 32,000 up to 768,000 samples. The latter very large number of samples may be considered redundant, given the acceptance fraction of $\simeq 0.35$ indicating an optimal output from the MCMC sampler (Foreman-Mackey et al. 2013).

The best-fitting models and their residuals are illustrated in the top and bottom panels of Figs. 4, 5 and 6, for A, $\mathrm{B}$ and $\mathrm{C}$ datasets, respectively. The posterior distribution is illustrated only for Dataset C (Fig. 7), since the posteriors for Datasets A and B are very similar, and therefore, to save space, we do not quote them. We note that the time of pericenter argument $\tau$, the binary period $P_{\text {bin }}$, and the timeshift $\Delta t_{0}$ from the cycle $L=0$ epoch are represented relative to the best-fitting parameters in Tab. 2, respectively, where $T_{0}=$ BJD 2455793.840061. These parameters are very close to the initial values derived with the common maximization of the likelihood function $\mathcal{L}$.

The posterior projections reveal relatively significant correlations of particular model parameters, like $(K, P)$ and $(P, \tau)$. However, the posterior is uni-modal with a quite strong peak. This is illustrated in Fig. 7 for a few selected parameters of the $(\mathrm{O}-\mathrm{C})$ model. The MCMC sampling reveals the error floor of $\simeq 2$ seconds for models optimal in the sense defined above. Without this correction, the "raw" reduced $\chi_{v}^{2} \sim 2$ indicates underestimated uncertainties. The optimal solution is represented with a red curve, and is overplotted on 100 randomly selected model curves from the MCMC sample. We found that the eccentricity of the best-fitting orbit $e \simeq 0.175$, indicating a significantly skewed $(\mathrm{O}-\mathrm{C})$ curve, and a relatively large semi-amplitude of the LTT signal $K \simeq 50 \mathrm{~s}$, rule out pericenter precession of the orbit, following Beuermann et al. (2012b).

Due to apparently random residuals with a rms $\simeq 10 \mathrm{sec}$ onds, which is almost equal to the mean of the rescaled uncertainties, we did not analyse models with additional parameters, such as the parabolic ephemeris (Hinse et al. 2014) or even a putative second companion (Almeida et al. 2013). The most simple, 1-companion model with the linear ephemeris, does not exhibit systematic, long-term changes of the $(\mathrm{O}-$ C) superimposed on the quasi-sinusoidal variation. Secular changes of the orbital period should not be expected for such a detached binary (Beuermann et al. 2012b).

To infer the companion mass from the third-body model parameters listed in Tab. 2, we used the stellar masses as $M_{1}=0.419 M_{\odot}$ and $M_{2}=0.109 M_{\odot}$ for the primary and the secondary, respectively, following Almeida et al. (2012). The best-fitting orbital period of $P \simeq 3600$ days implies the minimal mass of $\sim 15$ Jupiter masses (when the orbits are co-planar), that is in the brown dwarf. For the ratio of orbital periods $P / P_{\text {bin }} \sim 4 \times 10^{4}$, the triple system is highly hierarchical. Obviously, the brown dwarf has a stable orbit, which is two orders of magnitude wider than the stability limit $\simeq 0.2 a_{\text {bin }}$ (roughly $\sim 0.01$ au for the NSVS 14256825 binary) expected for circumbinary companions, if the binary eccentricity $e_{\text {bin }} \simeq 0$ (e.g., Holman \& Wiegert 1999, their Table 7). In such a case, the brown dwarf eccentricity $\sim 0.2$ has a negligible impact on the stability.

The third-body parameters determined here substantially differ from previous estimates. For instance, Beuermann et al. (2012b) reported the orbital period unconstrained between 20 and 70 years with eccentricity $e \simeq 0.50$ for a $20 \mathrm{yrs}$ orbit, since their data did not cover the $(\mathrm{O}-\mathrm{C})$ maximum re- 

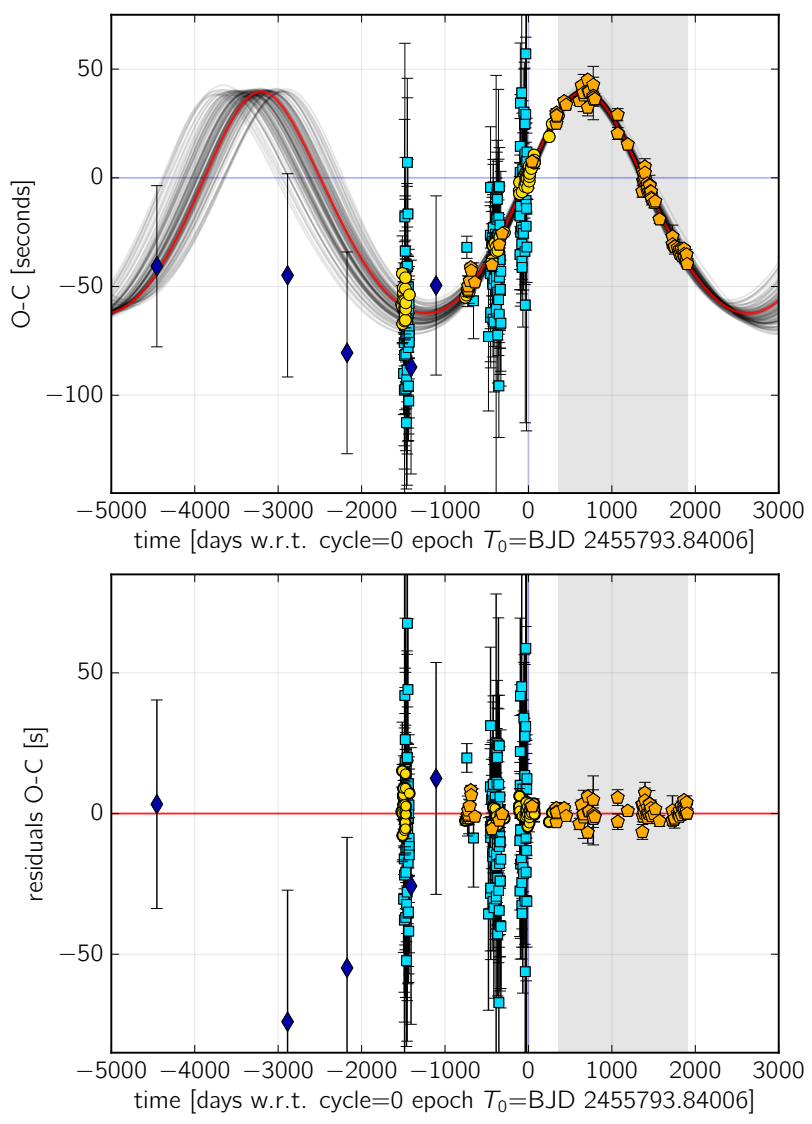

Figure 4. Top panel: the synthetic curve of the best-fitting model (red curve) to all timing data (Dataset A). Grey curves illustrate 100 randomly selected parameter samples from the MCMC posterior. Bottom panel: residuals to the best-fitting solution.

vealed here. We determine the semi-amplitude of the LTT signal being roughly twice larger than that stated in Hinse et al. (2014). The amplitude of $(\mathrm{O}-\mathrm{C})$ is one of crucial parameters needed to estimate the energy required to support the Applegate cycles (e.g., Völschow et al. 2016).

\section{DISCUSSION}

\subsection{The NSVS and ASAS timing data}

We would like to comment on the five earliest points from the literature, i.e. one timing measurement from the NSVS (Woźniak et al. 2004b) survey and four measurements from the ASAS survey (Pojmanski 1997), all five presented by Beuermann et al. (2012b).

It may be observed that these data exhibit large uncertainties and they strongly deviate from the models shown in this work. The exposure time is 80 seconds for NSVS survey, and as long as 180 seconds for the ASAS light curves. Moreover, to derive eclipses, one must collect measurements by folding photometric points spanning over a whole year.

Therefore we decided to re-analyse the source photometric data to derive the timings in an independent way. We
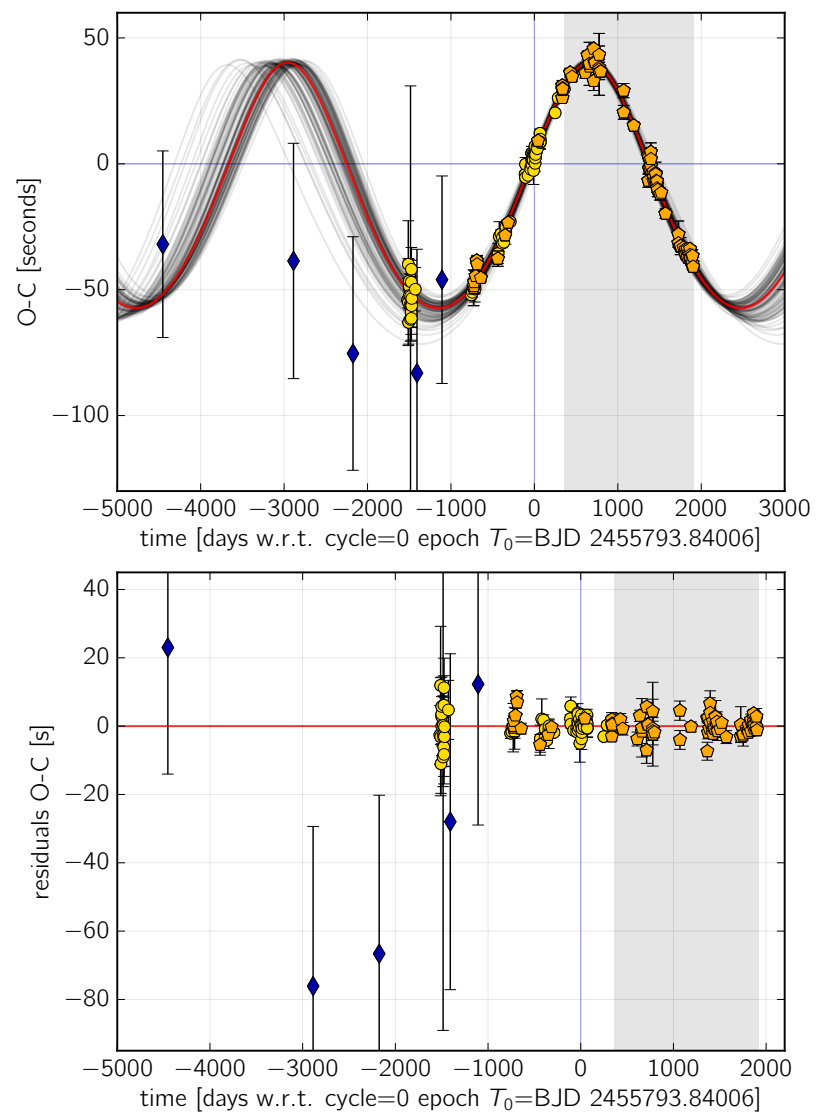

Figure 5. Top panel: the synthetic curve of the best-fitting model (red curve) to all data excluding SuperWASP data (Dataset B). Grey curves illustrate 100 randomly selected parameter samples from the MCMC posterior. Bottom panel: residuals to the best-fitting solution.

downloaded the source light curves from the publicly available NSVS ${ }^{1}$ and ASAS $^{2}$ archives. The NSVS light curve spans epochs between 1999 and 2000, while the ASAS observations span over a few years between 2003 and 2008. The photometric NSVS measurements timestamps are given in MJD UTC, while the ASAS measurements in HJD UTC. Therefore we recomputed the observation moments to the standard Solar system barycenter BJD time-scale with a procedure developed by Eastman et al. (2010). The data have been divided into $\sim 1$ year intervals and phase-folded with the orbital period of the binary derived from the most recent linear ephemeris. We know that this period is determined with an uncertainty to a few parts of millisecond, and assuming that the linear ephemeris is valid, we may use this new, fixed estimate.

In the next step we attempted to fit the function representing primary eclipses, as proposed in Beuermann et al.

\footnotetext{
${ }^{1}$ http://skydot.lanl.gov/

2 http://www.astrouw.edu.pl/asas/
} 

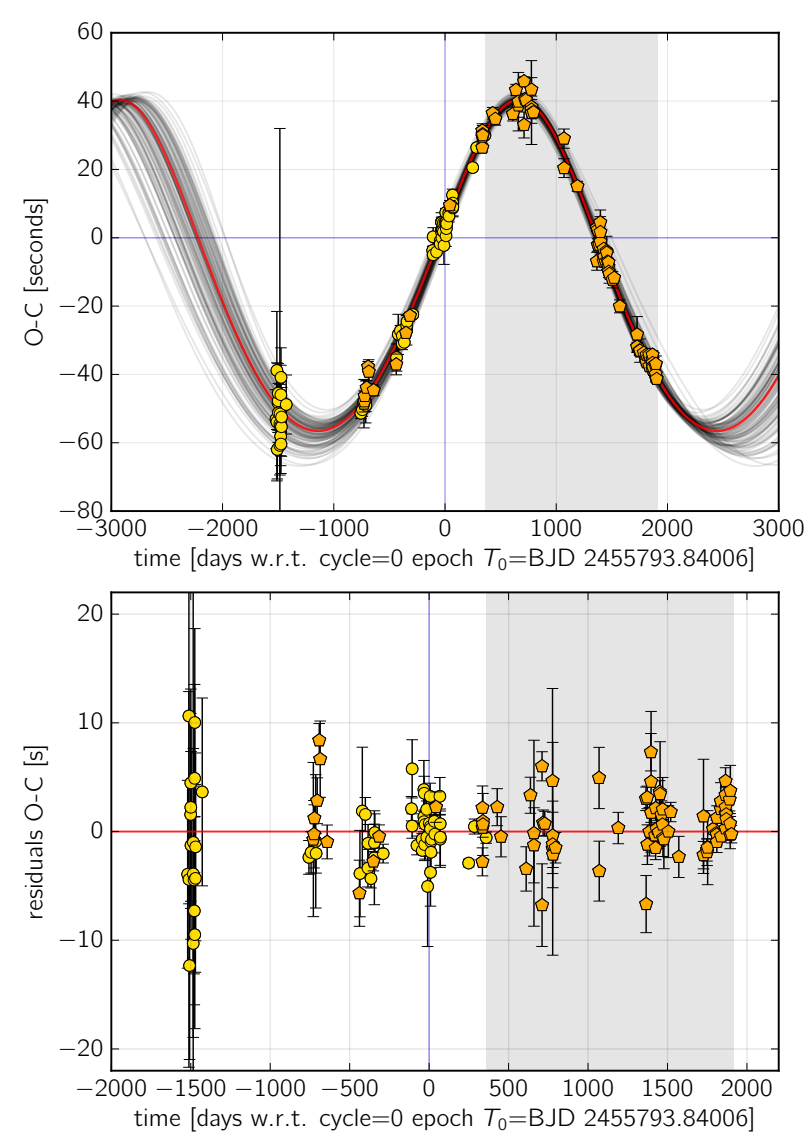

Figure 6. Top panel: the synthetic curve of the best-fitting model (red curve) to data without SuperWASP and NSVS/ASAS points (Dataset C). Grey curves illustrate 100 randomly selected parameter samples from the MCMC posterior. Bottom panel: residuals to the best-fitting solution displayed in Tab. 2 .

(2012b). We performed two experiments. In the first one, we fitted the whole set of parameters. In the second experiment, we constructed the mean, synthetic light-curve from the best 71 eclipses derived with the standard method. Then we fitted only some of the model eclipse parameters as free.

Unfortunately, all these experiments resulted in variable mid-eclipses estimates spread over 90 seconds. Moreover, for the ASAS data, the O-C of our timing moments are systematically $\sim 60$ second earlier than the measurements listed in Beuermann et al. (2012b).

The obtained formal errors from the model curve fits were on the level of $\pm 35-42$ seconds and are similar to the (OC) deviations from the linear ephemeris. Also the folding of photometric data for a year (which is around 3300 binary cycles) introduces a systematic shift of the mid-eclipses, in accord with the local $(\mathrm{O}-\mathrm{C})$ trend. It could be as large as $\sim 20-50$ seconds.

We conclude that the NSVS and ASAS mid-eclipse measurements are not very useful for the O-C analysis od such a short period binary. Fortunately, the parameters of our O-
$\mathrm{C}$ models derived for each of the three data-sets are similar. We believe that the final fit shown in Tab. 2, derived for Dataset $\mathrm{C}$, does indeed represent a reliable solution.

\subsection{The Applegate mechanism of the $(\mathrm{O}-\mathrm{C})$}

For compact binaries, like NSVS 14256825, magnetic activity of the less massive component may trigger Solar-like cycles and reshape the internal structure of this star (e.g., Applegate 1992; Lanza et al. 1998; Brinkworth et al. 2006). This leads to changes of the mutual gravitational field and oscillations of the orbital period. A common problem for this origin of the $(\mathrm{O}-\mathrm{C})$ variations is insufficient energy budget of the secondary required to change its quadrupole moment $Q$. Therefore, the Applegate cycles are usually dismissed in the literature as a possible explanation of cyclic variations of the $(\mathrm{O}-\mathrm{C})$ observed in a number of PCEBs. There are, however, more detailed and improved models of the Applegate mechanism, which modify the energy requirements (Lanza et al. 1998; Lanza 2006; Brinkworth et al. 2006). Recently, Völschow et al. (2016) considered a few variants of the Brinkworth et al. (2006) formulation that generally takes into account more realistic stellar density profiles. They analysed a sample of 15 compact PCEBs, including NSVS 14256825, and found that only for four systems in the sample, the magnetic cycles may be responsible for the (O-C) behaviour. For NSVS 14256825 the relative energy $\Delta E / E_{\mathrm{sec}}$ required to trigger the measured (O-C) should be between $\sim 5.4$ for the "classic" Applegate model and $\simeq 100$ for an advanced model of the stellar density profile (for a constant density profile, the ratio is 2 orders of magnitude larger, $\sim 3000$ ).

The up-to date $(\mathrm{O}-\mathrm{C})$ analysed in this paper implies a substantial change of the semi-amplitude $K$ and the variation period, we recalculated estimates of the energy budget $\Delta E / E_{\mathrm{sec}}$ for NSVS 14256825 given in Völschow et al. (2016). We recomputed this value in accord with their Eq. 7, following Tian et al. (2009), for canonical models in Applegate (1992) as well for the modified Applegate mechanism in Lanza et al. (1998); Lanza (2006, and references therein). We used also data for the secondary component from their Tab. 1.

Adopting the secondary radius $R_{2}=0.162 R_{\odot}$, mass $M_{2}=$ $0.109 M_{\odot}$, orbital separation $a=0.80 R_{\odot}$, and the effective temperature $T=2550 \mathrm{~K}$ we found that $\Delta E / E_{\mathrm{sec}} \simeq 11$. We computed the period change relative to the binary period

$$
\frac{\Delta P}{P_{\text {bin }}}=4 \pi \frac{K}{P} \simeq 2 \times 10^{-6},
$$

with the semi-amplitude $K \simeq 49 \mathrm{~s}$ and (O-C) oscillation $P \simeq 9.95 \mathrm{yrs}$ (modulation period) as displayed in Tab. 2. The quadrupole period variation $\Delta Q$ needed to drive the modulation of the orbital period (Lanza \& Rodonò 1999) is:

$$
\frac{\Delta P}{P_{\text {bin }}}=-9 \frac{\Delta Q}{M_{\text {bin }} a_{\text {bin }}^{2}},
$$




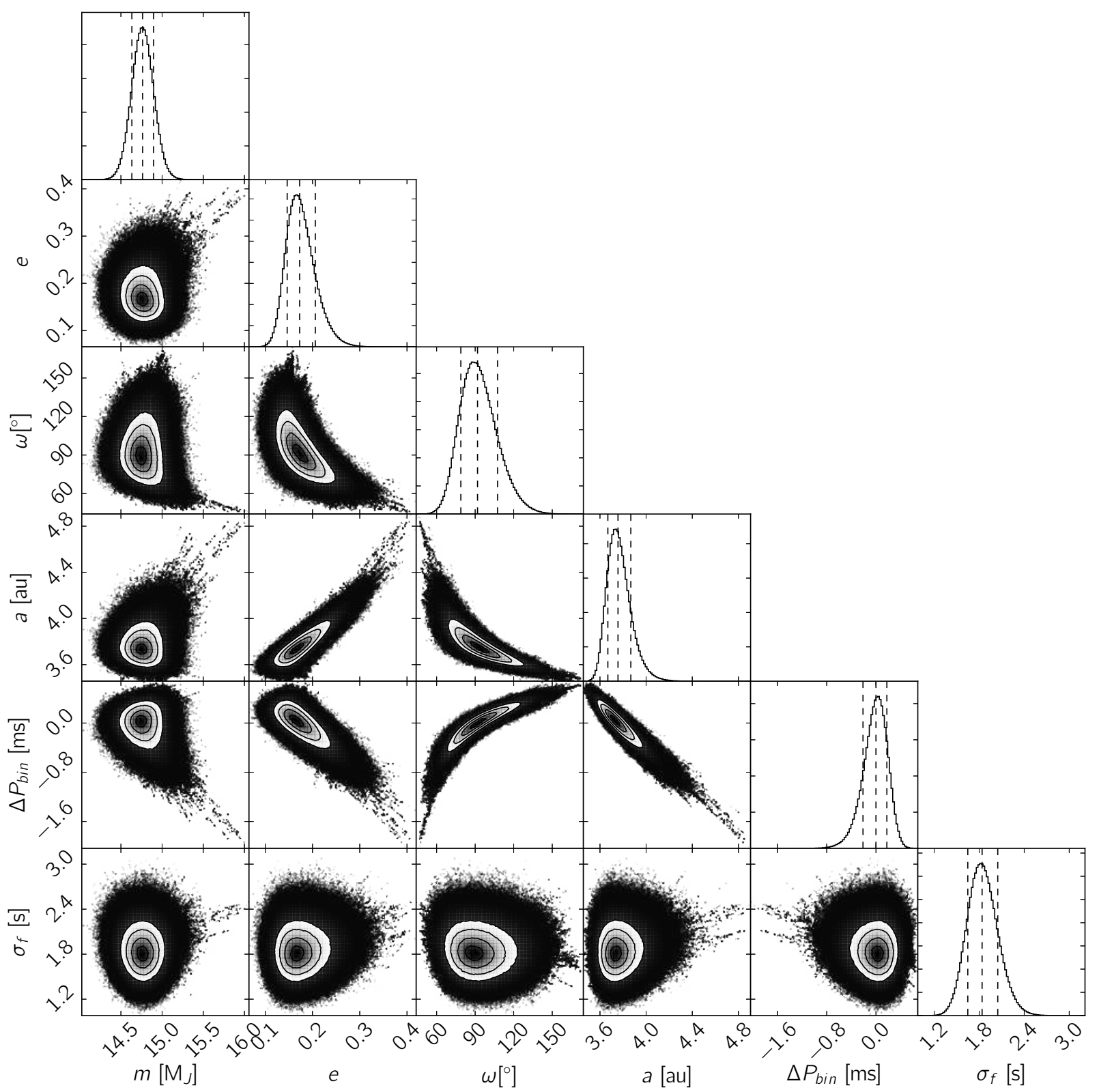

Figure 7. One- and two-dimensional projections of the posterior probability distributions of a few parameters inferred from the $(\mathrm{O}-\mathrm{C})$ model for Dataset C (Tab. 2 and Fig. 6). There are illustrated 32,000 samples for 512 "walkers" initiated in a small ball around the best-fitting model in Tab. 2. We removed about of $10 \%$ initial, burn-out samples. Subsequent parameters are the companion mass $m$, eccentricity $e$, periastron argument $\omega$, semi-major axis $a$, a deviation of the binary period $\Delta P_{\text {bin }}$ from its adopted best fitting value (in milliseconds, see Tab. 2), and the measurements uncertainty correction factor $\sigma_{f}$. Contours are for the 16th, 50th and 84th percentile of samples in the posterior distribution. See the text for details regarding parametrisation of the $(\mathrm{O}-\mathrm{C})$ model and imposed priors.

where $M_{\text {bin }}$ and $a_{\text {bin }}$ are the binary mass and the semi-major axis, respectively. For NSVS 14256825, we obtain the magnitude of $\Delta Q \simeq 10^{47} \mathrm{~g} \mathrm{~cm}^{2}$.

The updated $\Delta E / E_{\mathrm{sec}}$ is more than two times larger than the value in Völschow et al. (2016) for the genuine Applegate model which, in accord with their analysis, tends to underestimate the energy ratio. For other variants, based on the Brinkworth et al. (2006) formulation, and realistic stel- lar density profiles, the Applegate modulations are even less probable, since the prescribed energy budget is then by 1-2 orders of magnitude too small, as shown in Völschow et al. (2016, their Tab. 4).

In accord with the alternative generalisation of the Applegate mechanism by Lanza et al. (1998); Lanza (2006), taking into account additional factor of the Lorenz force, the magnetic cycles may operate with a fraction of the energy re- 
quired by the original Applegate model. Yet the lower limit of the calculated $\Delta E / E_{\mathrm{sec}} \sim 11$ factor even for this scenario seems to be so large that one can safely conclude that the Applegate mechanism and its generalizations proposed by Brinkworth et al. (2006), Lanza (2006) and Völschow et al. (2016) are not a credible explanations of the (O-C) variability in the NSVS 14256825 binary.

\section{CONCLUSIONS}

Our new set of light-curves of the NSVS 14256825 binary substantially extends the archived list of eclipse timing. For the first time our new data cover the maximum of the $(\mathrm{O}-\mathrm{C})$ w.r.t. the linear ephemeris, covering almost one full cycle of a quasi-sinusoidal modulation, and making it possible to put constrains on previous LTT models aimed at explaining the $(\mathrm{O}-\mathrm{C})$ behaviour of this system (Beuermann et al. 2012b; Almeida et al. 2013; Hinse et al. 2014).

In accord with the third-body hypothesis, the observed (OC) variations in the NSVS 14256825 may be explained by the presence of a single companion with a minimal mass in the brown dwarf mass range (14.7 Jupiter masses), in a moderately eccentric orbit with eccentricity $\simeq 0.2$, and the orbital period of $\sim 10$ years. We found that parameters of this thirdbody within our best model are relatively well constrained through the present data. The residuals do not indicate any significant secular trends which could appear due to dissipative phenomena in the binary (like mass transfer, magnetic braking and gravitational radiation). We note that Beuermann et al. (2012b) and Hinse et al. (2014) reported such trends due to much shorter observational window that did not cover the maximum of the $(\mathrm{O}-\mathrm{C})$ shown in this work.

Therefore, a 1-companion model may be the most reliable explanation of the NSVS 14256825 (O-C). Taking into account the updated amplitude of LTT of $K \simeq 50 \mathrm{~s}$ and its period of $\simeq 10 \mathrm{yr}$, the alternative hypothesis - the Applegate mechanism - does not seem to be sufficiently effective to produce such changes. In accord with a very recent analysis by Völschow et al. (2016), the energy required to trigger the Applegate cycles in the secondary companion should be 10-100 larger than its nuclear energy. Moreover, the relatively large $K$ and the the shape of $(\mathrm{O}-\mathrm{C})$ yielding the third-body orbit eccentricity of $\simeq 0.17$, rule out also the orbital precession as a plausible $(\mathrm{O}-\mathrm{C})$ variations mechanism.

The presence of a massive companion in moderately eccentric orbit around the evolved, compact binary would be not necessarily unusual on the grounds of the planet formation theory. Many scenarios are possible, regarding both first generation planets (companions) that survived the CommonEnvelope (CE) phase, as well as emerged in a protoplanetary disc formed from the stellar matter ejected during the $\mathrm{CE}$ phase, as second generation planets (e.g., Veras et al. 2011; Veras \& Tout 2012; Portegies Zwart 2013; Bear \&
Soker 2014; Völschow et al. 2014; Kostov et al. 2016a; Veras et al. 2017). In the first case, the best-fitting orbital elements of NSVS 14256825 may be used as the border conditions required to reconstruct the binary evolution, as shown by Portegies Zwart (2013).

We do not analyse other possibilities of the $(\mathrm{O}-\mathrm{C})$ variability, like the mass transfer, orbital precession, magnetic braking or gravitational radiation, which are usually refuted for this class of binaries.

We should stress, however, that the third-body hypothesis investigated for a number of close and evolved PCEBs reported in the literature, remains uncertain in most cases. A very discouraging example of this kind is the Cataclysmic Variable (CV) polar, HU Aqr (Schwope et al. 1993; Schwarz et al. 2009; Qian et al. 2011; Hinse et al. 2012; Goździewski et al. 2012; Schwope \& Thinius 2014; Bours et al. 2014; Goździewski et al. 2015). The apparently quasi-sinusiodal (O-C) variations with a full amplitude of $\sim 60 \mathrm{~s}$ observed for almost 20 years till 2012, has changed to a strong, secular trend that deviates by $180 \mathrm{~s}$ from the linear ephemeris to date. Two and three planet models of this system are strongly unstable, unless we consider an exotic system of three 5- $6 M_{\text {Jup }}$ mass planets, with the middle one revolving in a retrograde orbit. Such a system may be stable for at least 1 Gyr (Goździewski et al. 2015). Similarly, the (O-C) of HW Vir interpreted through 5:2 MMR configuration of two Jovian planets (Beuermann et al. 2012a) are not constrained, regarding the outermost planet and its mass. The $(\mathrm{O}-\mathrm{C})$ for other PCEBs, like NY Vir (Qian et al. 2012b; Lee et al. 2014), QS Vir (Horner et al. 2013), UZ For (Potter et al. 2011) could be formally explained with the resonant 2-planet systems, yet none of them has been found stable. An exception is the NN Ser with low-mass, Jovian planets close to 2:1 mean motion resonance, which is well documented nad has passed so far all tests of the planetary nature of the $(\mathrm{O}-\mathrm{C})$ (Beuermann et al. 2010; Marsh et al. 2014; Völschow et al. 2014). A few other PCEBs, like V471 Tau (Hardy et al. 2015), V470 Cam (Qian et al. 2013), RR Cae (Qian et al. 2012a) might host single-companions, see Almeida et al. (2013) and Zorotovic \& Schreiber (2013) listing such binaries with their astrophysical characteristics.

Observations of these systems is still timely since the longterm, hardly predictable (O-C) are typically known for a fraction of the longest putative orbital periods. There are open problems regarding the PCBEs, like the formation of putative companions as first- or second-generation planets, orbital architectures and stability of hypothetical multiple-companion configurations, the presence of mechanisms alternative or coexisting with the Applegate and Lanza-Rodonó cycles and the LTT effect. Therefore, while the planetary hypothesis of the (O-C) observed for NSVS 14256825 cannot be yet definite, our observations and new data may contribute more 
light on the unresolved astrophysical questions. For instance, the $(\mathrm{O}-\mathrm{C})$ amplitude constrains the energy required to trigger magnetic cycles of the M-dwarf component.

Additional, long-term timing observations of the NSVS 14256825 binary are required. Being relatively bright, the NSVS 14256825 system may be systematically monitored, as we show here, with $\sim 1 \mathrm{~m}$ class telescopes. During next 23 years, the third-body model and the eclipse ephemeris can be verified due to $(\mathrm{O}-\mathrm{C})$ approaching the nearby minimum (see Fig. 3 for our prediction).

\section{ACKNOWLEDGEMENTS}

We thank to the anonymous reviewer for his/her critical and informative comments which improved and this work. This work has been supported by The Scientific and Technological Research Council of Turkey (TUBITAK), through project number 114F460 (IN, AS, HE). This work has been supported by Polish National Science Centre grant DEC2011/03/D/ST9/00656 (AS, KK, MŻ). We thank the team of TUBITAK National Observatory (TUG) for a partial support in using T100 telescope with project number TUG T10063. We also wish to thank Adiyaman University Observatory (Turkey) and the Skinakas Observatory (Heraklion, Greece) teams. K.G. thanks the staff of the Poznan Supercomputer and Network Centre (PCSS) for the support and computational resources (grant 195).

Facility: Skinakas:1.3m

Software: Python, IRAF, SExtractor, emcee (ForemanMackey et al. 2013)

\section{APPENDIX: ADDITIONAL FIGURES AND TABLES}

\section{REFERENCES}

Almeida, L. A., Jablonski, F., \& Rodrigues, C. V. 2013, ApJ, 766, 11

Almeida, L. A., Jablonski, F., Tello, J., \& Rodrigues, C. V. 2012, MNRAS, 423, 478

Applegate, J. H. 1992, ApJ, 385, 621

Bear, E., \& Soker, N. 2014, MNRAS, 444, 1698

Beuermann, K., Dreizler, S., Hessman, F. V., \& Deller, J. 2012a, A\&A, 543, A138

Beuermann, K., Hessman, F. V., Dreizler, S., et al. 2010, A\&A, 521, L60

Beuermann, K., Buhlmann, J., Diese, J., et al. 2011, A\&A, 526, A53

Beuermann, K., Breitenstein, P., Debski, B., et al. 2012b, A\&A, 540, A8

Bours, M. C. P., Marsh, T. R., Breedt, E., et al. 2014, MNRAS, 445, 1924

Brinkworth, C. S., Marsh, T. R., Dhillon, V. S., \& Knigge, C. 2006, MNRAS, 365, 287

Charbonneau, P. 1995, ApJS, 101, 309

Deeg, H. J., Ocaña, B., Kozhevnikov, V. P., et al. 2008, A\&A, 480, 563

Doyle, L. R., Carter, J. A., Fabrycky, D. C., et al. 2011, Science, 333, 1602

Eastman, J., Siverd, R., \& Gaudi, B. S. 2010, PASP, 122, 935

Foreman-Mackey, D., Hogg, D. W., Lang, D., \& Goodman, J. 2013, PASP, 125, 306

Goodman, J., \& Weare, J. 2010, Comm. Apl. Math and Comp. Sci., 1,65

Goździewski, K., Nasiroglu, I., Słowikowska, A., et al. 2012, MNRAS, 425, 930

Goździewski, K., Słowikowska, A., Dimitrov, D., et al. 2015, MNRAS, 448, 1118
Hardy, A., Schreiber, M. R., Parsons, S. G., et al. 2015, ApJL, 800, L24

Hinse, T. C., Lee, J. W., Goździewski, K., et al. 2012, MNRAS, 420, 3609

Hinse, T. C., Lee, J. W., Goździewski, K., Horner, J., \& Wittenmyer, R. A. 2014, MNRAS, 438, 307

Holman, M. J., \& Wiegert, P. A. 1999, AJ, 117, 621

Horner, J., Hinse, T. C., Wittenmyer, R. A., Marshall, J. P., \& Tinney, C. G. 2012, MNRAS, 427, 2812

Horner, J., Wittenmyer, R. A., Hinse, T. C., et al. 2013, MNRAS, 435, 2033

Irwin, J. B. 1952, ApJ, 116, 211

Kilkenny, D., \& Koen, C. 2012, MNRAS, 421, 3238

Kostov, V. B., Moore, K., Tamayo, D., Jayawardhana, R., \& Rinehart, S. A. 2016a, ApJ, 832, 183

Kostov, V. B., Orosz, J. A., Welsh, W. F., et al. 2016b, ApJ, 827, 86 Lanza, A. F. 2006, MNRAS, 369, 1773

Lanza, A. F., \& Rodonò, M. 1999, A\&A, 349, 887

Lanza, A. F., Rodono, M., \& Rosner, R. 1998, MNRAS, 296, 893

Lee, J. W., Hinse, T. C., Youn, J.-H., \& Han, W. 2014, MNRAS, 445, 2331

Lee, J. W., Kim, S.-L., Kim, C.-H., et al. 2009, AJ, 137, 3181

Lohr, M. E., Norton, A. J., Anderson, D. R., et al. 2014, A\&A, 566, A128

Marsh, T. R., Parsons, S. G., Bours, M. C. P., et al. 2014, MNRAS, 437, 475

Pojmanski, G. 1997, AcA, 47, 467

Portegies Zwart, S. 2013, MNRAS, 429, L45

Potter, S. B., Romero-Colmenero, E., Ramsay, G., et al. 2011, MNRAS, 416, 2202

Pribulla, T., Vaňko, M., Ammler-von Eiff, M., et al. 2012, Astronomische Nachrichten, 333, 754 


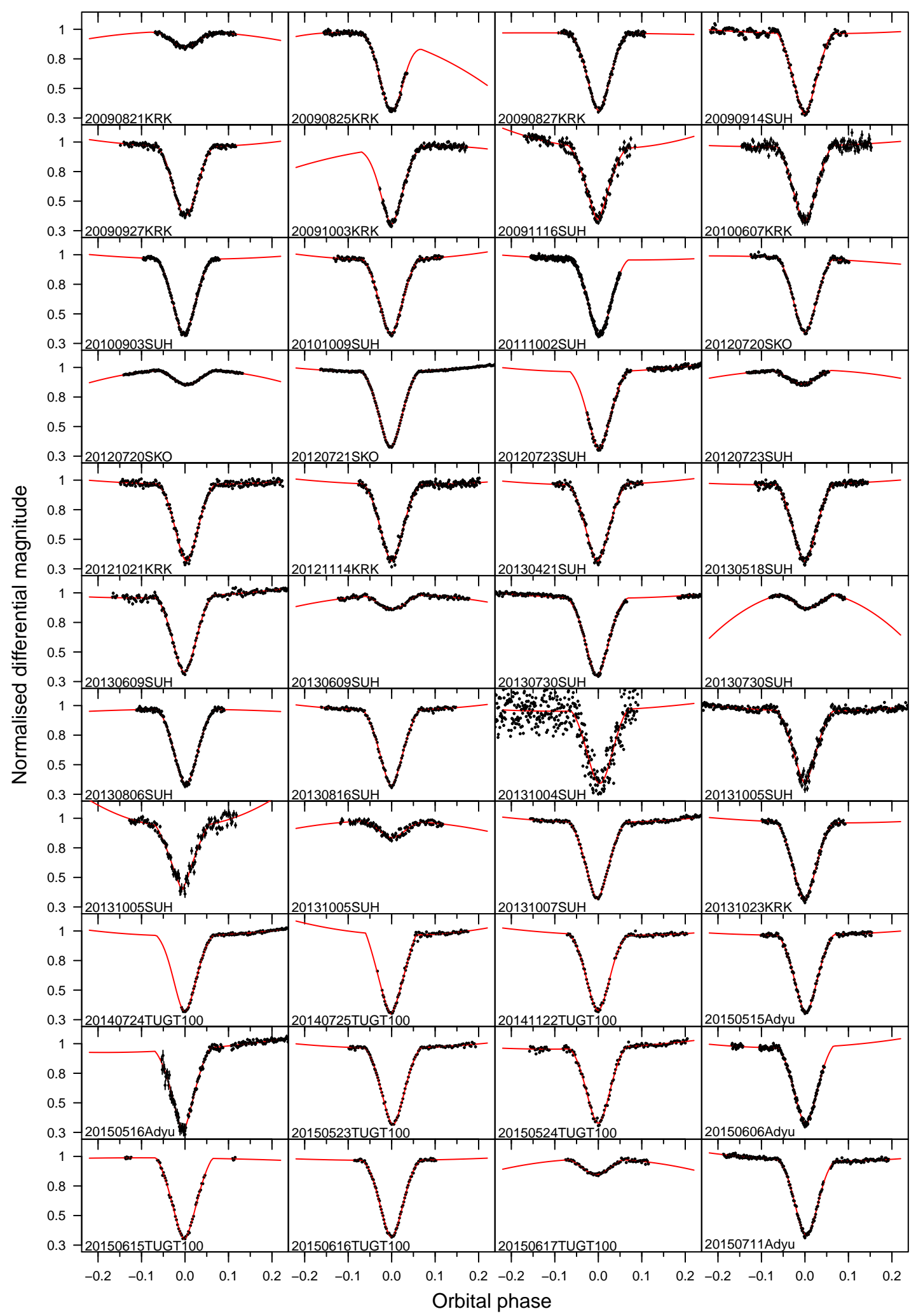

Figure 8. Primary and secondary eclipses of NSVS 14256825 from the 1-m telescope of the TUBITAK National Observatory (TUG), the 0.6-m telescope of the Adiyaman University Observatory (ADYU60), the 1.3-m telescope of the Skinakas Observatory (SKO), the 0.5-m telescope of the Astronomical Observatory of the Jagiellonian University (KRK) and the 0.6-m telescope of the Mt. Suhora Observatory (SUH), fitted with the equation as described in Section 2. of Beuermann et al. (2012b). 


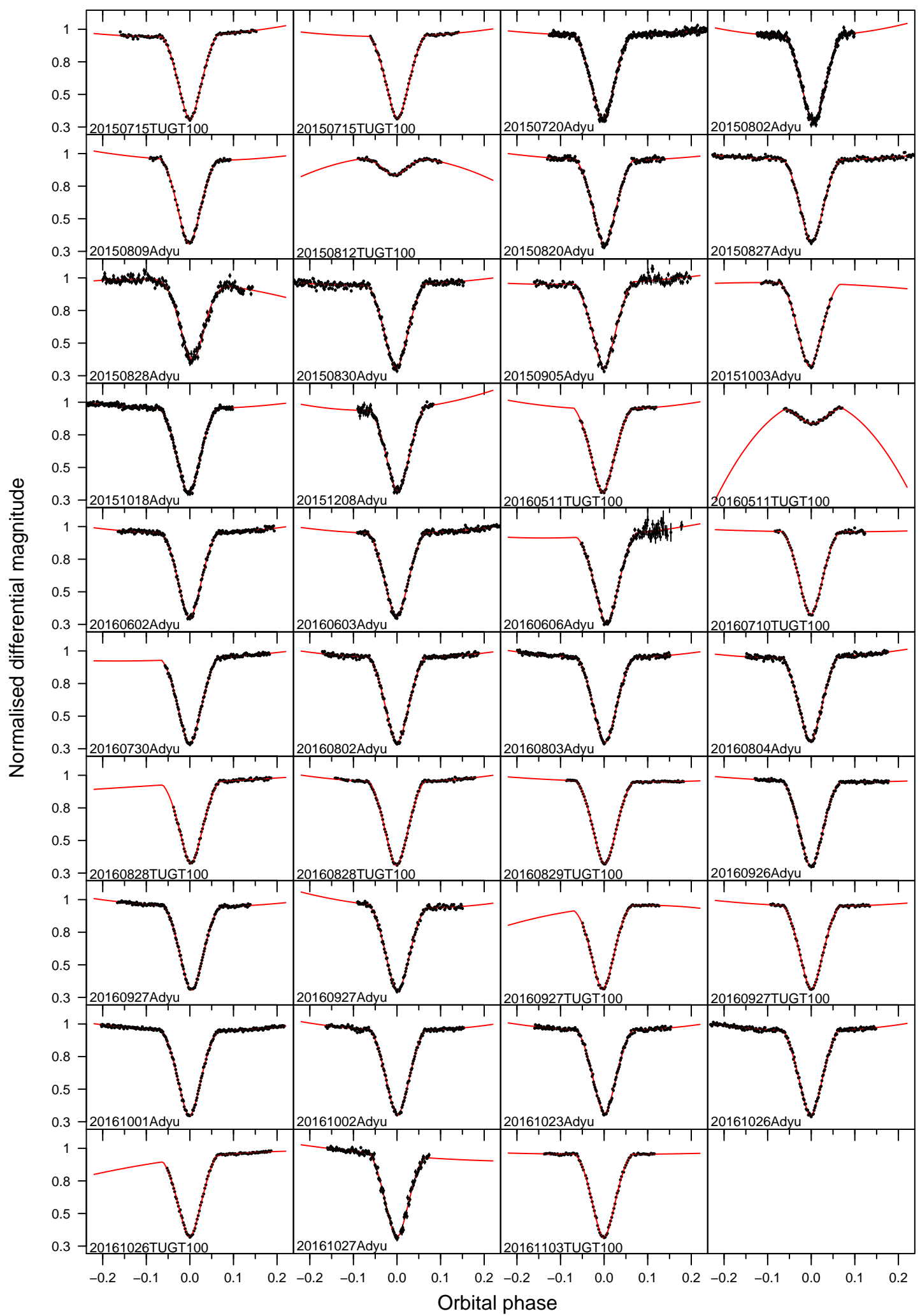

Figure 9. Primary and secondary eclipses of NSVS 14256825 from the 1-m telescope of the TUBITAK National Observatory (TUG), the 0.6-m telescope of the Adiyaman University Observatory (ADYU60), the 1.3-m telescope of the Skinakas Observatory (SKO), the 0.5-m telescope of the Astronomical Observatory of the Jagiellonian University (KRK) and the 0.6-m telescope of the Mt. Suhora Observatory (SUH), fitted with the equation as described in Section 2. of Beuermann et al. (2012b). 
Table 3. NSVS 14256825 observations log: starting date of observations, cycle, eclipse type ( 1 for primary, 2 for secondary), filter, exposure time, readout time and observatory code: KRK - the Astronomical Observatory of the Jagiellonian University, SUH - the Mt. Suhora Observatory, SKO - the Skinakas Observatory, TUG - the TUBITAK National Observatory, ADYU60 - the Adiyaman University Observatory. Exp $\equiv$ exposure time in seconds, $\mathrm{Rd} \equiv$ redout time in seconds, Obs. $\equiv$ observatory.

\begin{tabular}{|c|c|c|c|c|c|c|}
\hline Date & $L$ & Type & Filter & Exp & $\mathrm{Rd}$ & Obs. \\
\hline $2009-08-21$ & 7167.5 & 2 & BG40 & 10 & 2.2 & KRK \\
\hline $2009-08-25$ & 7204.0 & 1 & BG40 & 12 & 2.3 & KRK \\
\hline $2009-08-27$ & 7223.0 & 1 & BG40 & 10 & 2.2 & KRK \\
\hline 2009-09-14 & 7386.0 & 1 & W-light & 12 & 3.0 & SUH \\
\hline 2009-09-27 & 7503.0 & 1 & BG40 & 12 & 2.2 & KRK \\
\hline 2009-10-03 & 7557.0 & 1 & BG40 & 12 & 2.3 & KRK \\
\hline $2009-11-16$ & 7955.0 & 1 & W-light & 10 & 3.0 & SUH \\
\hline 2010-06-08 & 9797.0 & 1 & BG40 & 12 & 5.0 & KRK \\
\hline 2010-09-03 & 10593.0 & 1 & $\mathrm{R}$ & 8 & 2.8 & SUH \\
\hline 2010-10-09 & 10918.0 & 1 & $\mathrm{R}$ & 12 & 2.8 & SUH \\
\hline 2011-10-02 & 14162.0 & 1 & $\mathrm{R}$ & 5 & 2.4 & SUH \\
\hline 2012-07-20 & 16808.0 & 1 & $\mathrm{R}$ & 5 & 12.0 & SKO \\
\hline 2012-07-20 & 16808.5 & 2 & $\mathrm{R}$ & 5 & 12.0 & SKO \\
\hline 2012-07-21 & 16817.0 & 1 & $\mathrm{R}$ & 5 & 12.0 & SKO \\
\hline $2012-07-23$ & 16835.0 & 1 & BG40 & 10 & 2.4 & SUH \\
\hline $2012-07-23$ & 16835.5 & 2 & BG40 & 10 & 2.4 & SUH \\
\hline $2012-10-21$ & 17650.0 & 1 & BG40 & 12 & 2.0 & KRK \\
\hline 2012-11-14 & 17867.0 & 1 & BG40 & 10 & 2.0 & KRK \\
\hline 2013-04-22 & 19301.0 & 1 & $\mathrm{R}$ & 12 & 4.8 & SUH \\
\hline 2013-05-18 & 19545.0 & 1 & $\mathrm{R}$ & 10 & 2.4 & SUH \\
\hline 2013-06-09 & 19744.0 & 1 & W-light & 15 & 4.5 & SUH \\
\hline 2013-06-09 & 19744.5 & 2 & W-light & 15 & 4.5 & SUH \\
\hline 2013-07-30 & 20206.0 & 1 & W-light & 10 & 5.0 & SUH \\
\hline 2013-07-30 & 20206.5 & 2 & W-light & 10 & 5.0 & SUH \\
\hline 2013-08-06 & 20269.0 & 1 & $\mathrm{R}$ & 10 & 4.6 & SUH \\
\hline 2013-08-16 & 20360.0 & 1 & $\mathrm{R}$ & 15 & 4.8 & SUH \\
\hline 2013-10-04 & 20803.0 & 1 & $\mathrm{R}$ & 3 & 4.8 & SUH \\
\hline 2013-10-05 & 20812.0 & 1 & $\mathrm{R}$ & 10 & 3.1 & SUH \\
\hline 2013-10-05 & 20812.5 & 2 & $\mathrm{R}$ & 10 & 3.1 & SUH \\
\hline 2013-10-05 & 20813.0 & 1 & $\mathrm{R}$ & 10 & 3.1 & SUH \\
\hline 2013-10-07 & 20830.0 & 1 & $\mathrm{R}$ & 15 & 4.8 & SUH \\
\hline $2013-10-23$ & 20975.0 & 1 & BG40 & 12 & 3.0 & KRK \\
\hline 2014-07-24 & 23458.0 & 1 & W-light & 5 & 13.9 & TUG \\
\hline 2014-07-25 & 23467.0 & 1 & W-light & 5 & 13.9 & TUG \\
\hline 2014-11-22 & 24553.0 & 1 & W-light & 5 & 13.7 & TUG \\
\hline $2015-05-16$ & 26132.0 & 1 & W-light & 15 & 1.0 & ADYU60 \\
\hline $2015-05-16$ & 26141.0 & 1 & W-light & 15 & 1.0 & ADYU60 \\
\hline $2015-05-24$ & 26205.0 & 1 & W-light & 5 & 14.0 & TUG \\
\hline $2015-05-24$ & 26213.0 & 1 & W-light & 3 & 14.0 & TUG \\
\hline
\end{tabular}

Table 3. NSVS 14256825 Observations log: Tab.3 cont.

\begin{tabular}{|c|c|c|c|c|c|c|}
\hline Date & $L$ & ET & Filter & Exp. & $\mathrm{R}$ & Obs. \\
\hline 2015-06-06 & 26331.0 & 1 & W-light & 10 & 1.0 & ADYU60 \\
\hline $2015-06-16$ & 26413.0 & 1 & W-light & 5 & 13.9 & TUG \\
\hline 2015-06-17 & 26422.0 & 1 & W-light & 5 & 14.0 & TUG \\
\hline 2015-06-17 & 26422.5 & 2 & W-light & 7 & 14.1 & TUG \\
\hline 2015-07-11 & 26648.0 & 1 & W-light & 15 & 1.0 & ADYU60 \\
\hline $2015-07-15$ & 26683.0 & 1 & W-light & 5 & 20.0 & TUG \\
\hline $2015-07-15$ & 26684.0 & 1 & W-light & 5 & 20.0 & TUG \\
\hline $2015-07-21$ & 26730.0 & 1 & W-light & 10 & 1.0 & ADYU60 \\
\hline 2015-08-02 & 26846.0 & 1 & W-light & 10 & 1.0 & ADYU60 \\
\hline 2015-08-09 & 26911.0 & 1 & W-light & 15 & 2.0 & ADYU60 \\
\hline $2015-08-12$ & 26937.5 & 2 & W-light & 5 & 12.9 & TUG \\
\hline $2015-08-20$ & 27009.0 & 1 & W-light & 10 & 6.0 & ADYU60 \\
\hline $2015-08-27$ & 27073.0 & 1 & W-light & 15 & 5.0 & ADYU60 \\
\hline $2015-08-28$ & 27082.0 & 1 & W-light & 10 & 1.0 & ADYU60 \\
\hline $2015-08-30$ & 27099.0 & 1 & W-light & 16 & 1.0 & ADYU60 \\
\hline 2015-09-05 & 27154.0 & 1 & W-light & 15 & 5.0 & ADYU60 \\
\hline $2015-10-03$ & 27408.0 & 1 & W-light & 20 & 5.0 & ADYU60 \\
\hline $2015-10-18$ & 27543.0 & 1 & W-light & 10 & 1.0 & ADYU60 \\
\hline 2015-12-08 & 28004.0 & 1 & W-light & 15 & 1.0 & ADYU60 \\
\hline 2016-05-11 & 29411.5 & 2 & W-light & 7 & 14.0 & TUG \\
\hline $2016-05-12$ & 29412.0 & 1 & W-light & 7 & 14.1 & TUG \\
\hline 2016-06-02 & 29611.0 & 1 & W-light & 15 & 1.0 & ADYU60 \\
\hline 2016-06-03 & 29620.0 & 1 & W-light & 15 & 1.0 & ADYU60 \\
\hline 2016-06-06 & 29647.0 & 1 & W-light & 15 & 1.0 & ADYU60 \\
\hline 2016-07-11 & 29956.0 & 1 & W-light & 10 & 14.9 & TUG \\
\hline $2016-07-30$ & 30135.0 & 1 & W-light & 15 & 1.0 & ADYU60 \\
\hline 2016-08-02 & 30163.0 & 1 & W-light & 15 & 1.0 & ADYU60 \\
\hline 2016-08-03 & 30172.0 & 1 & W-light & 15 & 1.0 & ADYU60 \\
\hline 2016-08-04 & 30180.0 & 1 & W-light & 15 & 1.0 & ADYU60 \\
\hline $2016-08-28$ & 30397.0 & 1 & W-light & 7 & 13.7 & TUG \\
\hline $2016-08-28$ & 30399.0 & 1 & W-light & 7 & 13.7 & TUG \\
\hline 2016-08-29 & 30408.0 & 1 & W-light & 10 & 14.1 & TUG \\
\hline $2016-09-26$ & 30660.0 & 1 & W-light & 15 & 1.0 & ADYU60 \\
\hline $2016-09-27$ & 30669.0 & 1 & W-light & 15 & 1.0 & ADYU60 \\
\hline 2016-09-27 & 30669.0 & 1 & W-light & 10 & 14.1 & TUG \\
\hline $2016-09-27$ & 30670.0 & 1 & W-light & 15 & 1.0 & ADYU60 \\
\hline $2016-09-27$ & 30670.0 & 1 & W-light & 10 & 14.1 & TUG \\
\hline 2016-10-01 & 30705.0 & 1 & W-light & 15 & 1.0 & ADYU60 \\
\hline 2016-10-02 & 30714.0 & 1 & W-light & 15 & 1.0 & ADYU60 \\
\hline $2016-10-23$ & 30904.0 & 1 & W-light & 15 & 1.0 & ADYU60 \\
\hline $2016-10-26$ & 30931.0 & 1 & W-light & 10 & 14.1 & TUG \\
\hline $2016-10-26$ & 30931.0 & 1 & W-light & 15 & 1.0 & ADYU60 \\
\hline $2016-10-27$ & 30941.0 & 1 & W-light & 15 & 1.0 & ADYU60 \\
\hline 2016-11-03 & 31004.0 & 1 & W-light & 10 & 14.1 & TUG \\
\hline
\end{tabular}


Table 4. List of the NSVS 14256825 eclipse times from the literature as well as new measurements. The data point number, time of the minimum with its error and the type of the eclipse ( 1 for primary, 2 for secondary) and references are given. References correspond to the following papers: (1) Wils et al. (2007), (2) Beuermann et al. (2012b), (3) Kilkenny \& Koen (2012), (4) Almeida et al. (2013), (5) the Astronomical Observatory of the Jagiellonian University (this work), (6) the Mt. Suhora Observatory (this work), (7) the Skinakas Observatory (this work), (8) the TUBITAK National Obs. (this work), (9) the Adiyaman Univ. Obs. (this work). Data from Lohr et al. (2014) (see the astro-ph version) are not included in this Table. $\mathrm{T}$ is for eclipse type.

\begin{tabular}{|c|c|c|c|c|c|c|}
\hline & Cycle & BJD & Error[d] & $\operatorname{Err}[\mathrm{s}]$ & $\mathrm{T}$ & Ref. \\
\hline 1 & -26586.0 & 2451339.803273 & 0.000429 & 37 & 1 & 2 \\
\hline 2 & -12390.0 & 2452906.673899 & 0.000541 & 47 & 1 & 2 \\
\hline 3 & -5931.0 & 2453619.579776 & 0.000537 & 46 & 1 & 2 \\
\hline 4 & 0.0 & 2454274.208800 & 0.000100 & 9 & 1 & 1 \\
\hline 5 & 72.0 & 2454282.155900 & 0.000200 & 17 & 1 & 1 \\
\hline 6 & 73.0 & 2454282.266100 & 0.000200 & 17 & 1 & 1 \\
\hline 7 & 108.0 & 2454286.129100 & 0.000100 & 9 & 1 & 1 \\
\hline 8 & 172.0 & 2454293.193200 & 0.000100 & 9 & 1 & 1 \\
\hline 9 & 180.0 & 2454294.076200 & 0.000100 & 9 & 1 & 1 \\
\hline 10 & 181.0 & 2454294.186600 & 0.000100 & 9 & 1 & 1 \\
\hline 11 & 190.0 & 2454295.179900 & 0.000100 & 9 & 1 & 1 \\
\hline 12 & 316.0 & 2454309.087000 & 0.001000 & 86 & 1 & 1 \\
\hline 13 & 317.0 & 2454309.197300 & 0.000100 & 9 & 1 & 1 \\
\hline 14 & 325.0 & 2454310.080400 & 0.000100 & 9 & 1 & 1 \\
\hline 15 & 362.0 & 2454314.164200 & 0.000100 & 9 & 1 & 1 \\
\hline 16 & 380.0 & 2454316.150900 & 0.000100 & 9 & 1 & 1 \\
\hline 17 & 397.0 & 2454318.027400 & 0.000100 & 9 & 1 & 1 \\
\hline 18 & 406.0 & 2454319.020600 & 0.000100 & 9 & 1 & 1 \\
\hline 19 & 407.0 & 2454319.131200 & 0.000100 & 9 & 1 & 1 \\
\hline 20 & 443.0 & 2454323.104500 & 0.000100 & 9 & 1 & 1 \\
\hline 21 & 452.0 & 2454324.097900 & 0.000100 & 9 & 1 & 1 \\
\hline 22 & 832.0 & 2454366.040100 & 0.000100 & 9 & 1 & 1 \\
\hline 23 & 1018.0 & 2454386.569297 & 0.000569 & 49 & 1 & 2 \\
\hline 24 & 3737.0 & 2454686.676900 & 0.000477 & 41 & 1 & 2 \\
\hline 25 & 6914.0 & 2455037.335341 & 0.000018 & 2 & 1 & 2 \\
\hline 26 & 7037.0 & 2455050.911367 & 0.000022 & 2 & 1 & 2 \\
\hline 27 & 7167.5 & 2455065.315208 & 0.000082 & 7 & 2 & 5 \\
\hline 28 & 7204.0 & 2455069.343870 & 0.000036 & 3 & 1 & 5 \\
\hline 29 & 7223.0 & 2455071.440996 & 0.000014 & 1 & 1 & 5 \\
\hline 30 & 7304.0 & 2455080.381278 & 0.000071 & 6 & 1 & 2 \\
\hline 31 & 7322.0 & 2455082.368000 & 0.000021 & 2 & 1 & 2 \\
\hline 32 & 7386.0 & 2455089.432002 & 0.000024 & 2 & 1 & 6 \\
\hline 33 & 7503.0 & 2455102.345843 & 0.000018 & 2 & 1 & 5 \\
\hline 34 & 7557.0 & 2455108.306027 & 0.000041 & 4 & 1 & 5 \\
\hline 35 & 7955.0 & 2455152.234856 & 0.000018 & 2 & 1 & 6 \\
\hline
\end{tabular}

Table 4. continued...

\begin{tabular}{|c|c|c|c|c|c|c|}
\hline & Cycle & BJD & Error[d] & $\operatorname{Err}[\mathrm{s}]$ & $\mathrm{T}$ & Ref \\
\hline 36 & 9797.0 & 2455355.544034 & 0.000035 & 3 & 1 & 5 \\
\hline 37 & 9823.5 & 2455358.468971 & 0.000046 & 4 & 2 & 2 \\
\hline 38 & 9959.0 & 2455373.424740 & 0.000068 & 6 & 1 & 2 \\
\hline 39 & 10131.0 & 2455392.409097 & 0.000018 & 2 & 1 & 2 \\
\hline 40 & 10279.0 & 2455408.744420 & 0.000020 & 2 & 1 & 4 \\
\hline 41 & 10287.0 & 2455409.627440 & 0.000020 & 2 & 1 & 4 \\
\hline 42 & 10451.0 & 2455427.728770 & 0.000010 & 1 & 1 & 4 \\
\hline 43 & 10593.0 & 2455443.401924 & 0.000010 & 1 & 1 & 6 \\
\hline 44 & 10646.0 & 2455449.251760 & 0.000050 & 4 & 1 & 3 \\
\hline 45 & 10647.0 & 2455449.362150 & 0.000020 & 2 & 1 & 3 \\
\hline 46 & 10673.0 & 2455452.231890 & 0.000020 & 2 & 1 & 3 \\
\hline 47 & 10918.0 & 2455479.273564 & 0.000012 & 1 & 1 & 6 \\
\hline 48 & 11146.5 & 2455504.494050 & 0.000010 & 1 & 2 & 4 \\
\hline 49 & 12763.0 & 2455682.913998 & 0.000013 & 1 & 1 & 2 \\
\hline 50 & 12799.0 & 2455686.887451 & 0.000013 & 1 & 1 & 2 \\
\hline 51 & 12799.5 & 2455686.942699 & 0.000031 & 3 & 2 & 2 \\
\hline 52 & 13077.0 & 2455717.571460 & 0.000010 & 1 & 1 & 3 \\
\hline 53 & 13368.0 & 2455749.690361 & 0.000006 & 1 & 1 & 4 \\
\hline 54 & 13377.0 & 2455750.683717 & 0.000004 & 0 & 1 & 4 \\
\hline 55 & 13469.0 & 2455760.838180 & 0.000027 & 2 & 1 & 2 \\
\hline 56 & 13469.5 & 2455760.893396 & 0.000031 & 3 & 2 & 2 \\
\hline 57 & 13470.0 & 2455760.948549 & 0.000011 & 1 & 1 & 2 \\
\hline 58 & 13488.0 & 2455762.935315 & 0.000018 & 2 & 1 & 2 \\
\hline 59 & 13511.0 & 2455765.473867 & 0.000017 & 1 & 1 & 2 \\
\hline 60 & 13542.0 & 2455768.895489 & 0.000037 & 3 & 1 & 2 \\
\hline 61 & 13629.0 & 2455778.498061 & 0.000009 & 1 & 1 & 4 \\
\hline 62 & 13632.0 & 2455778.829154 & 0.000010 & 1 & 1 & 2 \\
\hline 63 & 13682.0 & 2455784.347812 & 0.000064 & 6 & 1 & 2 \\
\hline 64 & 13768.0 & 2455793.840061 & 0.000012 & 1 & 1 & 2 \\
\hline 65 & 13827.0 & 2455800.352168 & 0.000014 & 1 & 1 & 2 \\
\hline 66 & 13828.0 & 2455800.462510 & 0.000013 & 1 & 1 & 2 \\
\hline 67 & 13845.0 & 2455802.338869 & 0.000024 & 2 & 1 & 2 \\
\hline 68 & 13846.0 & 2455802.449197 & 0.000036 & 3 & 1 & 2 \\
\hline 69 & 13872.0 & 2455805.318959 & 0.000015 & 1 & 1 & 2 \\
\hline 70 & 13873.0 & 2455805.429322 & 0.000017 & 1 & 1 & 2 \\
\hline 71 & 13899.0 & 2455808.299065 & 0.000016 & 1 & 1 & 2 \\
\hline 72 & 14062.0 & 2455826.290080 & 0.000020 & 2 & 1 & 3 \\
\hline 73 & 14089.0 & 2455829.270170 & 0.000020 & 2 & 1 & 3 \\
\hline 74 & 14162.0 & 2455837.327516 & 0.000016 & 1 & 1 & 6 \\
\hline 75 & 14379.0 & 2455861.278730 & 0.000020 & 2 & 1 & 3 \\
\hline 76 & 14397.0 & 2455863.265420 & 0.000030 & 3 & 1 & 3 \\
\hline 77 & 14400.0 & 2455863.596559 & 0.000010 & 1 & 1 & 2 \\
\hline
\end{tabular}


Table 4. continued...

\begin{tabular}{|c|c|c|c|c|c|c|}
\hline & Cycle & BJD & Error[d] & $\operatorname{Err}[\mathrm{s}]$ & $\mathrm{T}$ & Ref. \\
\hline 78 & 14406.0 & 2455864.258790 & 0.000030 & 3 & 1 & 3 \\
\hline 79 & 16024.0 & 2456042.844216 & 0.000004 & 0 & 1 & 4 \\
\hline 80 & 16350.0 & 2456078.826240 & 0.000008 & 1 & 1 & 4 \\
\hline 81 & 16808.0 & 2456129.377577 & 0.000015 & 1 & 1 & 7 \\
\hline 82 & 16808.5 & 2456129.432822 & 0.000023 & 2 & 2 & 7 \\
\hline 83 & 16817.0 & 2456130.370981 & 0.000006 & 0 & 1 & 7 \\
\hline 84 & 16835.0 & 2456132.357723 & 0.000017 & 1 & 1 & 6 \\
\hline 85 & 16835.5 & 2456132.412907 & 0.000033 & 3 & 2 & 6 \\
\hline 86 & 17019.0 & 2456152.666554 & 0.000006 & 1 & 1 & 4 \\
\hline 87 & 17650.0 & 2456222.312685 & 0.000020 & 2 & 1 & 5 \\
\hline 88 & 17867.0 & 2456246.263846 & 0.000021 & 2 & 1 & 5 \\
\hline 89 & 19301.0 & 2456404.540320 & 0.000024 & 2 & 1 & 6 \\
\hline 90 & 19545.0 & 2456431.471682 & 0.000019 & 2 & 1 & 6 \\
\hline 91 & 19744.0 & 2456453.436075 & 0.000040 & 3 & 1 & 6 \\
\hline 92 & 19744.5 & 2456453.491275 & 0.000099 & 9 & 2 & 6 \\
\hline 93 & 20206.0 & 2456504.428991 & 0.000015 & 1 & 1 & 6 \\
\hline 94 & 20206.5 & 2456504.484030 & 0.000044 & 4 & 2 & 6 \\
\hline 95 & 20269.0 & 2456511.382498 & 0.000014 & 1 & 1 & 6 \\
\hline 96 & 20360.0 & 2456521.426538 & 0.000013 & 1 & 1 & 6 \\
\hline 97 & 20803.0 & 2456570.322299 & 0.000098 & 9 & 1 & 6 \\
\hline 98 & 20812.0 & 2456571.315608 & 0.000025 & 2 & 1 & 6 \\
\hline 99 & 20812.5 & 2456571.370781 & 0.000113 & 10 & 2 & 6 \\
\hline 100 & 20813.0 & 2456571.425973 & 0.000046 & 4 & 1 & 6 \\
\hline 101 & 20830.0 & 2456573.302321 & 0.000014 & 1 & 1 & 6 \\
\hline 102 & 20975.0 & 2456589.306568 & 0.000016 & 1 & 1 & 5 \\
\hline 103 & 23458.0 & 2456863.365367 & 0.000033 & 3 & 1 & 8 \\
\hline 104 & 23467.0 & 2456864.358634 & 0.000032 & 3 & 1 & 8 \\
\hline 105 & 24553.0 & 2456984.224844 & 0.000017 & 1 & 1 & 8 \\
\hline 106 & 26132.0 & 2457158.505405 & 0.000012 & 1 & 1 & 9 \\
\hline 107 & 26141.0 & 2457159.498659 & 0.000030 & 3 & 1 & 9 \\
\hline 108 & 26205.0 & 2457166.562708 & 0.000012 & 1 & 1 & 8 \\
\hline 109 & 26213.0 & 2457167.445649 & 0.000021 & 2 & 1 & 8 \\
\hline 110 & 26331.0 & 2457180.469794 & 0.000026 & 2 & 1 & 9 \\
\hline 111 & 26413.0 & 2457189.520546 & 0.000020 & 2 & 1 & 8 \\
\hline 112 & 26422.0 & 2457190.513847 & 0.000010 & 1 & 1 & 8 \\
\hline 113 & 26422.5 & 2457190.569067 & 0.000075 & 6 & 2 & 8 \\
\hline 114 & 26648.0 & 2457215.458344 & 0.000012 & 1 & 1 & 9 \\
\hline 115 & 26683.0 & 2457219.321420 & 0.000012 & 1 & 1 & 8 \\
\hline 116 & 26684.0 & 2457219.431837 & 0.000013 & 1 & 1 & 8 \\
\hline 117 & 26730.0 & 2457224.509018 & 0.000014 & 1 & 1 & 9 \\
\hline 118 & 26846.0 & 2457237.312397 & 0.000017 & 1 & 1 & 9 \\
\hline 119 & 26911.0 & 2457244.486749 & 0.000012 & 1 & 1 & 9 \\
\hline
\end{tabular}

Table 4. continued...

\begin{tabular}{|c|c|c|c|c|c|c|}
\hline & Cycle & BJD & Error[d] & $\operatorname{Err}[\mathrm{s}]$ & $\mathrm{T}$ & Ref. \\
\hline 120 & 26937.5 & 2457247.411658 & 0.000056 & 5 & 2 & 8 \\
\hline 121 & 27009.0 & 2457255.303376 & 0.000015 & 1 & 1 & 9 \\
\hline 122 & 27073.0 & 2457262.367301 & 0.000016 & 1 & 1 & 9 \\
\hline 123 & 27082.0 & 2457263.360683 & 0.000034 & 3 & 1 & 9 \\
\hline 124 & 27099.0 & 2457265.237010 & 0.000020 & 2 & 1 & 9 \\
\hline 125 & 27154.0 & 2457271.307578 & 0.000031 & 3 & 1 & 9 \\
\hline 126 & 27408.0 & 2457299.342580 & 0.000028 & 2 & 1 & 9 \\
\hline 127 & 27543.0 & 2457314.243090 & 0.000010 & 1 & 1 & 9 \\
\hline 128 & 28004.0 & 2457365.125453 & 0.000022 & 2 & 1 & 9 \\
\hline 129 & 29411.5 & 2457520.476902 & 0.000061 & 5 & 2 & 8 \\
\hline 130 & 29412.0 & 2457520.532048 & 0.000015 & 1 & 1 & 8 \\
\hline 131 & 29611.0 & 2457542.496481 & 0.000012 & 1 & 1 & 9 \\
\hline 132 & 29620.0 & 2457543.489845 & 0.000014 & 1 & 1 & 9 \\
\hline 133 & 29647.0 & 2457546.469948 & 0.000039 & 3 & 1 & 9 \\
\hline 134 & 29956.0 & 2457580.575537 & 0.000013 & 1 & 1 & 8 \\
\hline 135 & 30135.0 & 2457600.332481 & 0.000014 & 1 & 1 & 9 \\
\hline 136 & 30163.0 & 2457603.422952 & 0.000012 & 1 & 1 & 9 \\
\hline 137 & 30172.0 & 2457604.416311 & 0.000011 & 1 & 1 & 9 \\
\hline 138 & 30180.0 & 2457605.299328 & 0.000013 & 1 & 1 & 9 \\
\hline 139 & 30397.0 & 2457629.250499 & 0.000009 & 1 & 1 & 8 \\
\hline 140 & 30399.0 & 2457629.471256 & 0.000020 & 2 & 1 & 8 \\
\hline 141 & 30408.0 & 2457630.464585 & 0.000006 & 1 & 1 & 8 \\
\hline 142 & 30660.0 & 2457658.278862 & 0.000008 & 1 & 1 & 9 \\
\hline 143 & 30669.0 & 2457659.272248 & 0.000008 & 1 & 1 & 9 \\
\hline 144 & 30669.0 & 2457659.272263 & 0.000014 & 1 & 1 & 8 \\
\hline 145 & 30670.0 & 2457659.382587 & 0.000009 & 1 & 1 & 8 \\
\hline 146 & 30670.0 & 2457659.382609 & 0.000015 & 1 & 1 & 9 \\
\hline 147 & 30705.0 & 2457663.245692 & 0.000008 & 1 & 1 & 9 \\
\hline 148 & 30714.0 & 2457664.239053 & 0.000010 & 1 & 1 & 9 \\
\hline 149 & 30904.0 & 2457685.210139 & 0.000012 & 1 & 1 & 9 \\
\hline 150 & 30931.0 & 2457688.190200 & 0.000015 & 1 & 1 & 8 \\
\hline 151 & 30931.0 & 2457688.190212 & 0.000011 & 1 & 1 & 9 \\
\hline 152 & 30941.0 & 2457689.293987 & 0.000027 & 2 & 1 & 9 \\
\hline 153 & 31004.0 & 2457696.247504 & 0.000009 & 1 & 1 & 8 \\
\hline
\end{tabular}

Qian, S.-B., Liu, L., Zhu, L.-Y., et al. 2012a, MNRAS, 422, 24 Qian, S.-B., Zhu, L.-Y., Dai, Z.-B., et al. 2012b, ApJL, 745, L23 Qian, S.-B., Zhu, L.-Y., Liu, L., et al. 2010, Ap\&SS, 329, 113 Qian, S.-B., Liu, L., Liao, W.-P., et al. 2011, MNRAS, 414, L16 Qian, S.-B., Shi, G., Zola, S., et al. 2013, MNRAS, 436, 1408 
Ribas, I. 2005, in Astronomical Society of the Pacific Conference Series, Vol. 335, The Light-Time Effect in Astrophysics: Causes and cures of the O-C diagram, ed. C. Sterken, 55

Schwarz, R., Schwope, A. D., Vogel, J., et al. 2009, A\&A, 496, 833

Schwope, A. D., \& Thinius, B. D. 2014, Astronomische Nachrichten, 335, 357

Schwope, A. D., Thomas, H. C., \& Beuermann, K. 1993, A\&A, 271, L25

Tian, Y. P., Xiang, F. Y., \& Tao, X. 2009, Ap\&SS, 319, 119

Veras, D., Georgakarakos, N., Dobbs-Dixon, I., \& Gänsicke, B. T. 2017, MNRAS, 465, 2053

Veras, D., \& Tout, C. A. 2012, MNRAS, 422, 1648

Veras, D., Wyatt, M. C., Mustill, A. J., Bonsor, A., \& Eldridge, J. J. 2011, MNRAS, 417, 2104
Völschow, M., Banerjee, R., \& Hessman, F. V. 2014, A\&A, 562, A19

Völschow, M., Schleicher, D. R. G., Perdelwitz, V., \& Banerjee, R. 2016, A\&A, 587, A34

Wils, P., di Scala, G., \& Otero, S. A. 2007, Information Bulletin on Variable Stars, 5800

Wittenmyer, R. A., Horner, J., \& Marshall, J. P. 2013, MNRAS, 431,2150

Woźniak, P. R., Williams, S. J., Vestrand, W. T., \& Gupta, V. 2004a, AJ, 128, 2965

Woźniak, P. R., Vestrand, W. T., Akerlof, C. W., et al. 2004b, AJ, 127,2436

Zhu, L., Qian, S., Liu, L., et al. 2011, in Astronomical Society of the Pacific Conference Series, Vol. 451, 9th Pacific Rim Conference on Stellar Astrophysics, ed. S. Qain, K. Leung, L. Zhu, \& S. Kwok, 155

Zorotovic, M., \& Schreiber, M. R. 2013, A\&A, 549, A95 